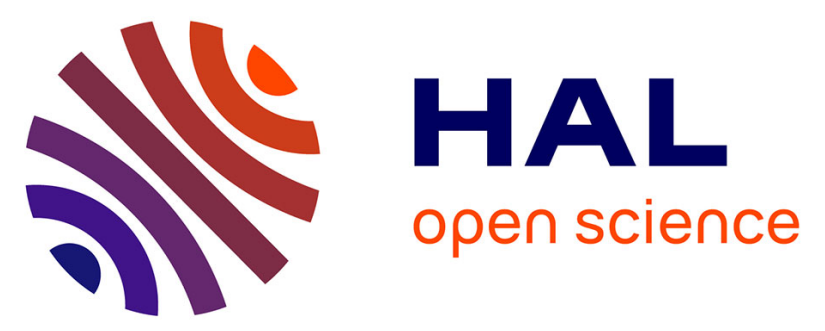

\title{
Stratosphere-troposphere coupling and annular mode variability in chemistry-climate models
}

E. P. Gerber, M. P. Baldwin, H. Akiyoshi, J. Austin, Slimane Bekki, P. Braesicke, N. Butchart, M. Chipperfield, M. Dameris, S. Dhomse, et al.

\section{- To cite this version:}

E. P. Gerber, M. P. Baldwin, H. Akiyoshi, J. Austin, Slimane Bekki, et al.. Stratosphere-troposphere coupling and annular mode variability in chemistry-climate models. Journal of Geophysical Research: Atmospheres, 2010, 115 (D3), pp.D00M06. 10.1029/2009JD013770 . hal-00510752

\section{HAL Id: hal-00510752 \\ https://hal.science/hal-00510752}

Submitted on 15 Jul 2020

HAL is a multi-disciplinary open access archive for the deposit and dissemination of scientific research documents, whether they are published or not. The documents may come from teaching and research institutions in France or abroad, or from public or private research centers.
L'archive ouverte pluridisciplinaire HAL, est destinée au dépôt et à la diffusion de documents scientifiques de niveau recherche, publiés ou non, émanant des établissements d'enseignement et de recherche français ou étrangers, des laboratoires publics ou privés. 


\title{
Stratosphere-troposphere coupling and annular mode variability in chemistry-climate models
}

\author{
Edwin P. Gerber, ${ }^{1}$ Mark P. Baldwin, ${ }^{2}$ Hideharu Akiyoshi, ${ }^{3}$ John Austin, ${ }^{4}$ Slimane Bekki, ${ }^{5}$ \\ Peter Braesicke, ${ }^{6}$ Neal Butchart, ${ }^{7}$ Martyn Chipperfield, ${ }^{8}$ Martin Dameris, ${ }^{9}$ Sandip Dhomse, ${ }^{8}$ \\ Stacey M. Frith, ${ }^{10,11}$ Rolando R. Garcia, ${ }^{12}$ Hella Garny, ${ }^{9}$ Andrew Gettelman, ${ }^{12}$ \\ Steven C. Hardiman, ${ }^{7}$ Alexey Karpechko, ${ }^{13}$ Marion Marchand, ${ }^{5}$ Olaf Morgenstern, ${ }^{14}$ \\ J. Eric Nielsen, ${ }^{11,15}$ Steven Pawson, ${ }^{15}$ Tom Peter, ${ }^{16,17}$ David A. Plummer, ${ }^{18,19}$ \\ John A. Pyle, ${ }^{6}$ Eugene Rozanov, ${ }^{16,17}$ John F. Scinocca, ${ }^{19}$ Theodore G. Shepherd, ${ }^{20}$ \\ and Dan Smale ${ }^{14}$ \\ Received 29 December 2009; revised 28 April 2010; accepted 5 May 2010; published 22 September 2010.
}

[1] The internal variability and coupling between the stratosphere and troposphere in CCMVal-2 chemistry-climate models are evaluated through analysis of the annular mode patterns of variability. Computation of the annular modes in long data sets with secular trends requires refinement of the standard definition of the annular mode, and a more robust procedure that allows for slowly varying trends is established and verified. The spatial and temporal structure of the models' annular modes is then compared with that of reanalyses. As a whole, the models capture the key features of observed intraseasonal variability, including the sharp vertical gradients in structure between stratosphere and troposphere, the asymmetries in the seasonal cycle between the Northern and Southern hemispheres, and the coupling between the polar stratospheric vortices and tropospheric midlatitude jets. It is also found that the annular mode variability changes little in time throughout simulations of the 21 st century. There are, however, both common biases and significant differences in performance in the models. In the troposphere, the annular mode in models is generally too persistent, particularly in the Southern Hemisphere summer, a bias similar to that found in CMIP3 coupled climate models. In the stratosphere, the periods of peak variance and coupling with the troposphere are delayed by about a month in both hemispheres. The relationship between increased variability of the stratosphere and increased persistence in the troposphere suggests that some tropospheric biases may be related to stratospheric biases and that a well-simulated stratosphere can improve simulation of tropospheric intraseasonal variability.

Citation: Gerber, E. P., et al. (2010), Stratosphere-troposphere coupling and annular mode variability in chemistry-climate models, J. Geophys. Res., 115, D00M06, doi:10.1029/2009JD013770.

\section{Introduction}

[2] The Northern and Southern Annular Modes (NAM and SAM) are the dominant patterns of variability in the

\footnotetext{
${ }^{1}$ Center for Atmosphere Ocean Science, Courant Institute of Mathematical Sciences, New York University, New York, New York, USA.

${ }^{2}$ NorthWest Research Associates, Redmond, Washington, USA.

${ }^{3}$ National Institute for Environmental Studies, Tsukuba, Japan.

${ }^{4}$ GFDL, University of Princeton, Princeton, New Jersey, USA.

${ }^{5}$ LATMOS, IPSL, UVSQ, UPMC, INSU, CNRS, Paris, France.

${ }^{6}$ University of Cambridge, Cambridge, UK.

${ }^{7}$ Met Office Hadley Centre, Exeter, UK.

${ }^{8}$ Institute for Climate and Atmospheric Science, University of Leeds, Leeds, UK.

${ }^{9}$ Deutsches Zentrum für Luft- und Raumfahrt, Institut für Physik der Atmosphäre, Oberpfaffenhofen, Germany.

Copyright 2010 by the American Geophysical Union. 0148-0227/10/2009JD013770
}

extratropical atmosphere on intraseasonal to interdecadal time scales [Thompson and Wallace, 2000]. The annular modes describe a coupled pattern of variability between the stratosphere and troposphere, characterizing the connection

\footnotetext{
${ }^{10}$ Atmospheric Chemistry and Dynamics Branch, NASA GSFC, Greenbelt, Maryland, USA.

${ }^{11}$ Space Science Applications Incorporated, Laurel, Maryland, USA.

${ }^{12}$ NCAR, Boulder, Colorado, USA.

${ }^{13}$ Finnish Meteorological Institute, Helsinki, Finland.

${ }^{14}$ National Institute of Water and Atmospheric Research, Lauder, New Zealand.

${ }^{15}$ Global Modeling and Assimilation Office, NASA GSFC, Greenbelt, Maryland, USA.

${ }^{16}$ PMOD, WRC, Zurich, Switzerland.

${ }^{17}$ IAC, ETH, Zurich, Switzerland.

${ }^{18}$ Environment Canada, Montreal, Quebec, Canada.

${ }^{19}$ Environment Canada, Victoria, British Columbia, Canada.

${ }^{20}$ Department of Physics, University of Toronto, Toronto, Ontario, Canada.
} 
between the position of the midlatitude, eddy driven jets in the troposphere and the strength of the polar night jets in the stratosphere. In this study, we use the annular modes to evaluate the ability of chemistry-climate models (CCMs) to capture the internal variability of the extratropical atmosphere. The CCM integrations were contributed to the second Chemistry-Climate Model Validation Activity (CCMVal-2) organized by SPARC to provide predictions of ozone loss and recovery for the $2010 \mathrm{WMO} / \mathrm{UNEP}$ assessment of ozone loss [Eyring et al., 2008; Morgenstern et al., 2010b]. To simulate ozone chemistry and its impact on the circulation, CCMs are run with increased vertical resolution, model tops above the stratopause, and more sophisticated stratospheric gravity wave parameterizations than most atmosphere-ocean climate prediction models (i.e., those considered in the Intergovernmental Panel on Climate Change Fourth Assessment Report, IPCC AR4), and so represent the state of the art in modeling the coupled troposphere-stratosphere system. They thus provide an ideal system to diagnose the influence of the stratosphere on the tropospheric circulation.

[3] To assess the coupling between the stratosphere and troposphere, we require a multilevel index with high time resolution that can be applied to both observational and model output [Baldwin and Thompson, 2009]. Care must be taken, particularly in the case of model integrations of future climate, to ensure that the metric remains focused on the internal variability of the atmosphere, and is not influenced by externally forced trends in the climatology. In section 3 we outline modifications to the Baldwin and Thompson [2009] procedure to compute the annular modes independently at each pressure level, with the goal of computing an index that remains true to the spirit of Thompson and Wallace [1998], but that can be applied to long climate prediction runs. After establishing a robust procedure, we show that the annular mode variability is remarkably insensitive to climate trends in model predictions of the 21 st century.

[4] The annular modes allow us to assess both the spatial and temporal structure of extratropical variability in the CCMs. While the gross spatial structure of the annular mode is quite generic, largely a consequence of the conservation of angular momentum and the mean structure of the jets [e.g., Gerber and Vallis, 2005; Wittman et al., 2005], details on the position and amplitude of the patterns provide stricter tests. We show that CCMs do fairly well, capturing tropospheric variability with equal fidelity and stratospheric variability with greater accuracy than CMIP3 models. Biases in the annular mode structure, however, reflect deficiencies with the CCM climatologies.

[5] As explored by Baldwin et al. [2003] and Graversen and Christiansen [2003], the seasonal and vertical structure of the annular mode variance, characteristic time scale, and the correlation between troposphere and stratosphere differs between the two hemispheres in a surprising way: active coupling in the Northern Hemisphere peaks during the boreal winter, while the Southern Hemisphere coupling peaks in the austral spring and summer. The temporal structure of the annular modes has been shown to involve eddy-mean flow feedbacks [e.g., Robinson, 1996; Lorenz and Hartmann, 2001; Gerber and Vallis, 2007; Son et al., 2008], synoptic wave breaking and high-latitude blocking [e.g., Benedict et al., 2004; Woollings et al., 2008; Strong and Magnusdottir, 2008] and interaction between the troposphere and stratosphere [e.g., Baldwin and Dunkerton, 2001; Wittman et al., 2004; Thompson et al., 2006; Gerber and Polvani, 2009], and so provides a challenging test of model fidelity. We show that the CCMs capture the key features of variability in both hemispheres, but that common biases across most models still exist, particularly in the timing of the Northern Hemisphere seasonal cycle and the persistence of variability in the Southern Hemisphere. The consistency in biases in the stratosphere and troposphere, however, suggests active coupling between the layers.

[6] The remainder of the paper is structured as follows: in section 2, we describe the reanalysis and model data used in this analysis. The procedure for computing the annular mode patterns and time series is described in section 3. Our results are presented in section 4 , and a discussion and our conclusions follow in sections 5 and 6.

\section{Data}

[7] The primary observational data used are daily mean geopotential height from the ECMWF (European Centre for Medium-Range Weather Forecasting) ERA-40 Reanalysis (September 1957 to August 2002) and ECMWF Operational analyses (September 2002 to December 2008), as described in the work of $\mathrm{Lu}$ et al. [2009]. Both data sets extend to $1 \mathrm{hPa}(\sim 50 \mathrm{~km})$. The ERA-40 Reanalysis has a spectral resolution of $\mathrm{T} 159$, corresponding to a $1.125^{\circ}$ horizontal resolution in latitude and longitude. The data are available at 23 standard pressure surfaces from $1000 \mathrm{hPa}$ to $1 \mathrm{hPa}$, which were assimilated using direct radiosonde and satellite measurements [Uppala et al., 2005]. The ECMWF Operational data were output from the ongoing analyses produced by the most recent ECMWF Integrated Forecasting System (IFS) model. Data from September 2002 to 2008 are available on the same $1.125^{\circ}$ grid and on 21 pressure levels, which are identical to the ERA-40 data except without the 600 and $775 \mathrm{hPa}$ levels. For consistency, only the data for those 21 pressure levels are used herein. Because observations were inadequate in the Southern Hemisphere prior to the widespread availability of satellite data, we restrict the ECMWF data there to the period 1979-2008. Where appropriate, we also include calculations based on NCEP-NCAR (National Centers for Environmental Prediction-National Center for Atmospheric Research) reanalyses [Kalnay et al., 1996] of zonal mean geopotential height from the same time period. NCEP-NCAR reanalyses have $2.5^{\circ}$ spatial resolution on 17 pressure levels up to $10 \mathrm{hPa}$. In general, there is very good agreement between the two reanalyses, so that results based on ECMWF output are quantitatively comparable to results based on NCEP-NCAR output.

[8] SPARC has established the Chemistry-Climate Model Validation Activity (CCMVal) for coupled chemistry-climate models (CCMs) [Eyring et al., 2005]. The goal of CCMVal is to improve understanding of chemistry-climate models and their underlying GCMs through process-oriented evaluation, along with discussion and coordinated analysis of science results [Eyring et al., 2010]. For this study, we use three types of CCMVal integrations [Eyring et al., 2008]. REF-B1 reference simulations were forced by observations from 1960 to 2006, representing the best estimate of the observed past. REF-B1 simulations have been designed to address primarily internal modeling uncertainties since SSTs, 
Table 1. CCMVal-2 Model Output Used in This Study ${ }^{\mathrm{a}}$

\begin{tabular}{lccc}
\hline \multicolumn{1}{c}{ Model } & REF-B0 & REF-B1 & REF-B2 \\
\hline AMTRAC3 & - & 1 & 1 \\
CCSRNIES & 1 & 1 & 1 \\
CMAM & 1 & 3 & 3 \\
E39CA & - & 1 & - \\
GEOSCCM & 1 & 1 & 1 \\
LMDZrepro & 1 & 1 & 1 \\
Niwa SOCOL & 1 & 1 & - \\
SOCOL & 1 & 3 & 3 \\
UMSLIMCAT & - & 1 & $1^{\mathrm{b}}$ \\
UMUKCA-METO & 1 & 1 & 2 \\
WACCM & - & 2 &
\end{tabular}

\footnotetext{
${ }^{\mathrm{a}}$ The number in each cell shows the number of ensemble runs for each scenario that were analyzed

${ }^{\mathrm{b}}$ The last complete year of integration in this REF-B2 simulation is 2083, and data from 2019 were missing.
}

sea ice, and external forcing such as volcanic eruptions and variations of solar irradiation are prescribed based on observations. REF-B2 reference simulations are forced by trace gas projections and modeled sea surface temperatures for 1960-2100, and so provide future predictions. With the exception of CMAM, which was coupled to a dynamical ocean, REF-B2 simulations include external uncertainties, because SST and sea ice data are obtained from climate simulations. Where available, we also used output from the 20 yearlong REF-B0 reference integrations, which are forced with annual repeating conditions representative of the year 2000 , and no externally forced variability. These integrations would provide an excellent environment to study the internal variability of the atmosphere, but were too short to be analyzed rigorously on their own. A list of all integrations used in this analysis is found in Table 1.

[9] Output from three coupled climate models in the World Climate Research Programme's (WCRP) Coupled Model Intercomparison Project phase 3 (CMIP3) multimodel data set were also assessed. These three models were the only CMIP3 models that provided daily zonal winds to $10 \mathrm{hPa}$, and so provide a small sample of the stratospheric variability in the CMIP3 data set. We use output from the 20C3M control simulations of 20th century climate from 1961 to 2000. Details on the models and forcing scenario can be found in the work of Meehl et al. [2007].

\section{Methods}

[10] Nearly all of our results are based on annular mode indices, and include many of the diagnostics of Baldwin and Dunkerton [1999, 2001] and Baldwin et al. [2003]. We have refined the methods used in those papers to define the annular modes in order to account for (1) data and model output extending to $1 \mathrm{hPa}$ and (2) the changing background climatology during the 1960-2100 REF-B2 model runs. It is also necessary to define a methodology that allows for consistent comparisons between data and models. We begin with the procedure detailed in the work of Baldwin and Thompson [2009]. They defined the annular modes, separately for each pressure level and hemisphere, as the leading EOF of daily, zonally averaged geopotential anomalies. This method is simpler to use than previous methods that relied on latitude-longitude maps of geopotential [e.g., Baldwin and Dunkerton, 2001], and is actually more robust, especially in the upper troposphere. The method requires only daily zonal mean geopotential, which was included as standard output in CCMVal-2 specifically so that the annular modes could be calculated. As daily geopotential height was not available from integrations of the CMIP3 models or the CCM AMTRAC3, it was estimated from daily zonal mean zonal wind, assuming geostrophic balance as in the work of Gerber et al. [2008a].

[11] Modifications to the Baldwin and Thompson [2009] methodology pertain to how the geopotential anomalies are defined as deviations from the climatology. Our first change relates back to the original annular mode concept of Thompson and Wallace [1998]. At pressure levels well above the surface, heating or cooling of the atmosphere, as, for example, after a volcanic eruption, can lead to global changes in geopotential height. In Thompson and Wallace's original conception of the annular mode, however, the pattern represents a north-south movement of mass, which can in turn be related to changes in the distribution of zonal momentum through geostrophic balance. To prevent global geopotential height fluctuations, which are disconnected from the zonal momentum structure, from aliasing onto the annular modes, we simply subtract the global mean geopotential from each day of data at each pressure level. This guarantees that the annular mode at each pressure level is defined such that the mass displacement over the polar cap is equal and opposite to that at lower latitudes, so reflecting meridional shifts of mass and momentum. It has no effect at the surface, where the global mean geopotential reflects the mass of the atmosphere, and very little effect below $5 \mathrm{hPa}$. Removing the global mean geopotential height also helps to remove secular trends in height driven by global temperature changes in the atmosphere. As documented below, however, trends in height exhibit considerable latitudinal variation, necessitating our second change to the procedure.

[12] The second modification regards the definition of the seasonally varying climatology, against which anomalies are defined. Most previous studies [e.g., Thompson and Wallace, 1998; Baldwin and Dunkerton, 2001] used a fixed seasonally varying climatology to define anomalies. This allows the annular mode indices to have trends, but the trends depend, in part, on how closely changes in the geopotential match the annular mode patterns. Figure 1a illustrates the $100 \mathrm{hPa} \mathrm{Z}$ climatology for 1960-1999 and 2060-2099 for CMAM, which is typical of the CCMs. We choose the $100 \mathrm{hPa}$ level because the trends are most extreme just above the tropopause. The geopotential height trend in the tropics is much larger than at high latitudes, due to increased warming in the tropics. Figure $1 \mathrm{~b}$ illustrates the difference between these two climatologies, compared with the NAM and SAM EOF patterns at $100 \mathrm{hPa}$ computed three different ways: (1) using the Baldwin and Thompson [2009] procedure applied to the 140 year REF-B2 integration, and ( 2 and 3 ) using the new procedure outlined below, where we remove global mean geopotential height and allow for a slowly varying seasonal cycle, applied to the 47 year REF-B1 and 140 year REF-B2 integrations, respectively.

[13] With the new procedure, the EOFs based on both REF-B1 and REF-B2 records are nearly identical, and match the observed patterns shown in Figure 4 quite well. The patterns computed when the Baldwin and Thompson [2009] 


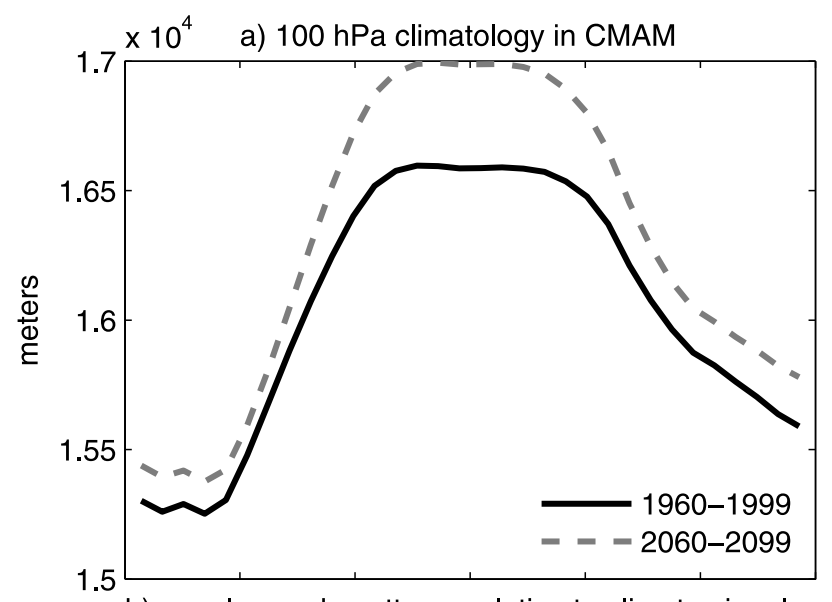

b) annular mode patterns relative to climate signal

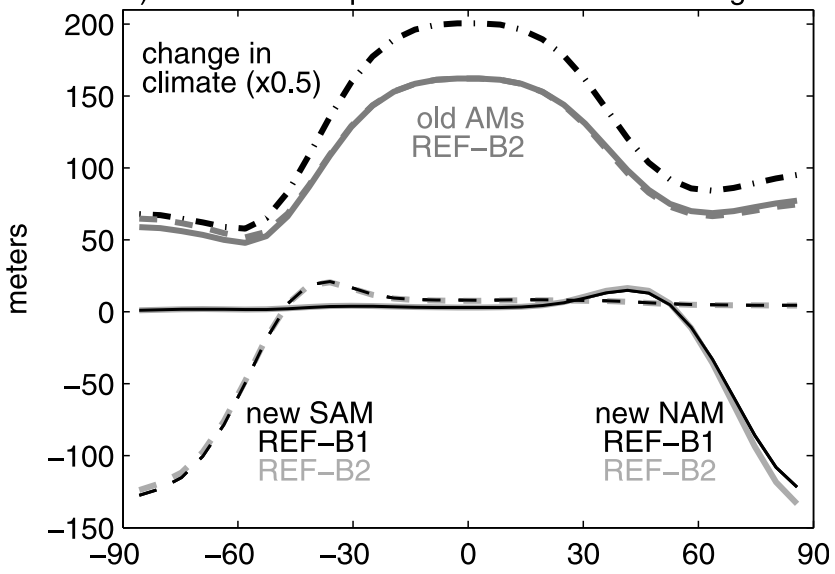

Figure 1. (a) The annual mean climatology of height $Z$ in CMAM, based on the last four decades of the 20th and 21st centuries. (b) The climate change signal (black, dash-dotted) determined as the difference between the two climatologies above and scaled by a factor of 0.5 , in comparison with the annular mode EOFs computed three different ways. In medium gray, the upper curves marked by "old AMs," the EOFs are computed from the full 140 year REF-B2 run with the [Baldwin and Thompson, 2009] procedure, where a fixed seasonally varying climatology is removed. For the black and light gray curves marked as "new NAM" and "new SAM," we use the revised procedure proposed in this paper: the black curves are based on the 47 year long REF-B1 integration, the light gray is the full 140 year REF-B2 integration. In each case, the NAM is solid and the SAM is dashed. As discussed in the text, the REF-B2 annular mode computed with the standard procedure is radically different, reflecting the trend and not the internal variability. With the new procedure, the annular mode always reflects the internal variability and is not affected by changes in the climate during the integration.

procedure is applied to the long REF-B2 integrations, however, are fundamentally different: the patterns based on the two hemispheres are almost the same, and almost perfectly follow the trend. The problem is that the structure of these EOFs changes radically to reflect the trend, rather than the internal variability. In the long integration, the trend explains more variance than the internal variability, and EOF analysis merely seeks to find patterns that maximize the variance. As the trend is global in scale, the annular mode in both hemispheres is the same.

[14] The key issue is how to compute EOFs based on data sets of differing lengths, as the strength of secular trends relative to the internal variability is a function of the length of the data set. In prior studies based on shorter ( $<50$ years) or historic data sets, secular trends were always relatively small and did not affect the EOF structure. When long data sets are used, however, one must be careful to define the annular mode patterns and indices in such a way that they always reflect internal variability. McLandress and Shepherd [2009] and Morgenstern et al. [2010a] address this concern in long integrations (similar CCMVal-2 REF-B2 simulations as considered here) by computing the NAM relative to shorter 40 year periods at the beginning and/or end of the integration. To establish a more general solution, we define a variable seasonal climatology that accounts for slowly varying trends driven by external climate forcing, trends that may vary as a function of season. We adopt an approach similar to that used by J. F. Scinocca (A nonparametric additive model analysis for multimodel time series, submitted to Journals of Geophysical Research, 2010) in which the term "trend" refers to a smooth trajectory passing through the time series data, leaving a "noise" field as the residual. Through direct experimentation, we found that the results are not sensitive to the details of how the slowly varying trend is defined, but that it is important to consider nonlinear trends, particularly to account for the formation and recovery of the ozone hole in the Southern Hemisphere.

[15] We illustrate the procedure in Figure 2. In the top, we plot the geopotential height anomaly relative to a fixed seasonal cycle at $100 \mathrm{hPa}$ and $41.5^{\circ} \mathrm{S}$ on 15 December of each year from 1960 to 2099 in a REF-B2 simulation of CMAM. This location falls in the midlatitude lobe of the SAM, and this calendar date was chosen to highlight the impact of ozone loss and recovery. The steady increase in height driven by greenhouse gas warming dominates the amplitude of internal variability in this long run, explaining the radical shift in the annular mode pattern in Figure $1 \mathrm{~b}$ in the case in which trends were not taken into account. Removing the global mean geopotential height before defining the seasonal cycle, we obtain the anomalies shown in Figure $2 b$. This filters out much of the global warming signal, but there are still low-frequency trends associated with known external forcings: the increase in height at this latitude relative to the global mean reflects the formation of the ozone hole from 1960 to 2000 . After this time, the height falls, reflecting both ozone recovery and the decrease in height in the midlatitudes relative to the tropics associated with climate change, as can be seen in Figure 1b. To prevent these slow trends from influencing our analysis of the natural variability, we compute a slowly varying trend climatology, $\bar{Z}_{T}(t, y, z)$, as shown by the gray line in Figure $2 \mathrm{~b}$. Anomalies defined relative to the slowly evolving climatology, which more accurately reflect the internal variability, are shown in the bottom.

[16] The slowly varying trend climatology, $\bar{Z}_{T}(t, y, z)$ must account for the seasonality of trends, but remove as little of the natural variability as possible. To prevent overfitting, we 


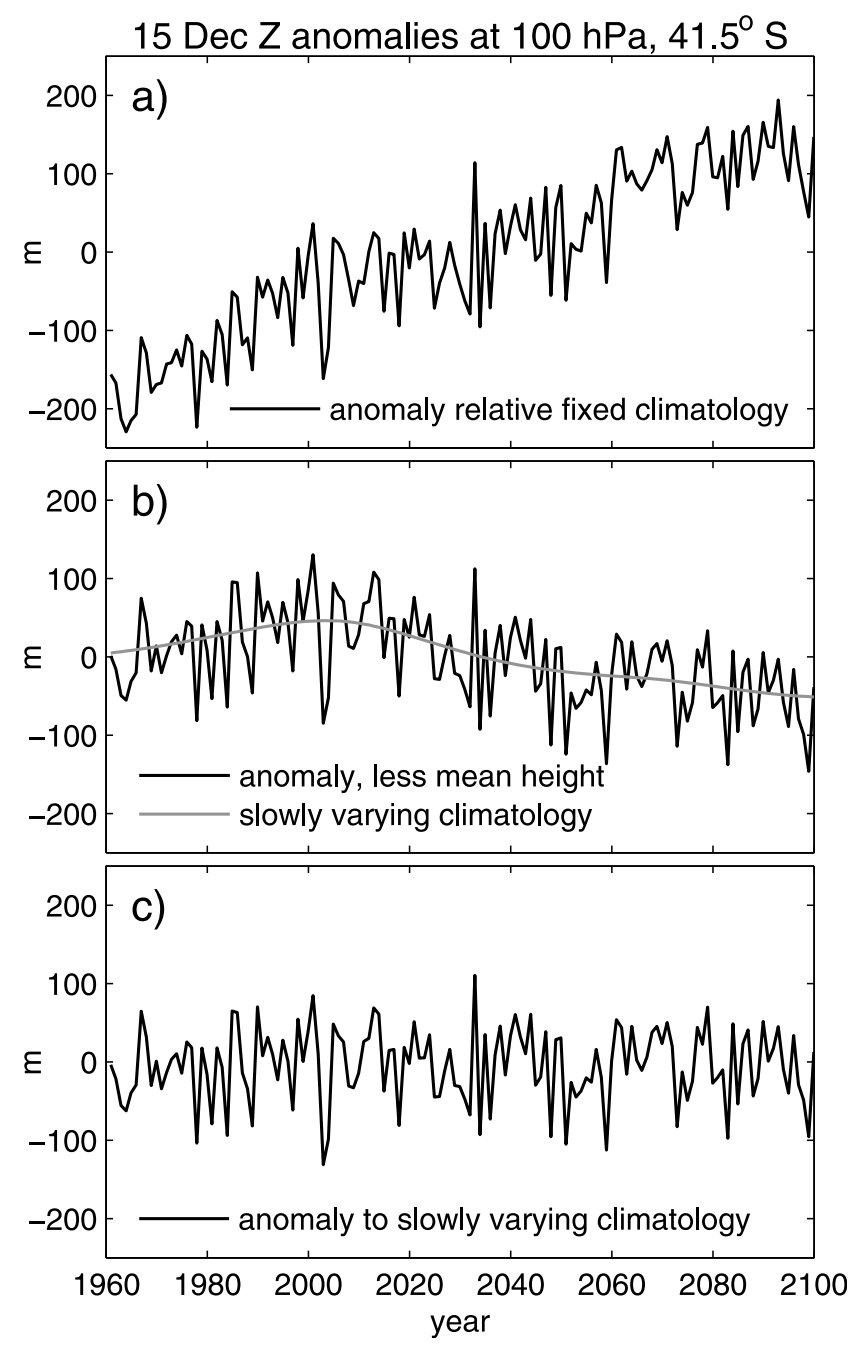

Figure 2. Geopotential height $(Z)$ anomalies at $100 \mathrm{hPa}$ and $41.5^{\circ} \mathrm{S}$, sampled once each year on 15 December from a 140 year REF-B2 integration of CMAM. 15 December is chosen to highlight the impact of the ozone hole. (a) Anomalies are defined relative to a fixed seasonal cycle, as is typically done in a standard EOF analysis, leaving a pronounced positive trend in geopotential height. (b) The global mean geopotential height has been removed at each date, in addition to a fixed seasonal cycle. This removes much of the global warming signal, but some externally forced variability associated with ozone loss and recovery and greenhouse gas induced heating remains, as captured by the gray curve. (c) The anomalies relative to this slowly varying climatology.

first applying a 60 day low pass filter to the daily zonal mean geopotential $\bar{Z}(t, y, z)$. Then, for each date and location, we apply a 30 year low pass filter to the smoothed time series, using only that calendar date of each year in the data set. This removes slowly varying trends at each given day. The initial smoothing regularizes the interannual low pass filter, so that the trend varies slowly throughout the year; we also experimented by computing trends first, and then smoothing, and obtained virtually identical results. The internal variability is the "noise:" $\bar{Z}_{I}(t, y, z)=\bar{Z}(t, y, z)-\bar{Z}_{T}(t, y, z)$. EOFs based on anomalies from the slowly varying trend are no longer sensitive to the length of the data set, as shown in Figure 1b. For shorter records, however, the impact is minimal. With the ECMWF reanalyses, we compared the annular mode time series computed as in the work of Baldwin and Thompson [2009] with the procedure here. In the Northern Hemisphere $(\mathrm{NH})$, the correlation is above 0.985 at all pressure levels below $3 \mathrm{hPa}$. It falls to 0.71 at $1 \mathrm{hPa}$, due in part to trends in the observation record and the impact of global mean height variation on the AM patterns. In the Southern Hemisphere $(\mathrm{SH})$, the correlation is greater than 0.986 at all levels up to $20 \mathrm{hPa}$. It falls to 0.95 by $3 \mathrm{hPa}$, and 0.67 at $1 \mathrm{hPa}$ for the same reasons as in the $\mathrm{NH}$.

[17] An additional advantage of removing a slowly varying climatology is that the resulting annular mode indices have no trends, and therefore can be used to compare variances, time scales, etc. that reflect only the internal variability. The trend information is retained in the variable climatology, and can be projected onto the patterns to obtain long-term trends in the index, as in the work of Morgenstern et al. [2010a]. As seen in Figure 1b, however, the NAM and SAM trends will only partially reflect the overall trend in geopotential due to poor overlap between the trends and natural modes of variability.

[18] To summarize, we compute the annular mode at each pressure level from the daily geopotential field as follows. The global mean geopotential is removed for each day, followed by the slowly varying seasonal climatology $\bar{Z}_{T}(t, y, z)$. The remaining anomaly field is then weighted by the square root cosine latitude, to account for the convergence of meridians toward the pole [North et al., 1982], and the NAM and SAM are calculated as the first EOF of anomalies between the equator and the respective pole. The daily annular mode index is then computed by projecting the anomaly field onto the EOF pattern, and normalized to have zero mean and unit variance. The annular mode pattern is then computed by regressing the anomaly fields onto the normalized time series; hence the amplitude of the variability is characterized by the spatial pattern. The observed annular mode spatial patterns were calculated from ERA-40 and NCEP-NCAR reanalysis data for the period 1958 or 1979 to 2008 , for the NH and SH, respectively. For the models, we define the annular mode pattern of each model based on one REF-B1 integration for the period 1960-2006. (With this new methodology, however, the EOF patterns computed from the various ensemble members and scenario integrations of a given model are quite similar, as seen in Figure $1 \mathrm{~b}$, and the results are not sensitive to which data set is used to compute the annular mode.) The annular mode indices for the REF-B0 and B2 scenario integrations, or additional REF-B1 ensemble members, were computed by projecting anomalies onto the standard annular mode pattern of the model, using the same normalization constants as computed from the control REF-B1 integration.

\section{Results}

[19] Baldwin and Thompson [2009] have shown that the annular mode provides an ideal multilevel index for assessing the coupling between the troposphere and stratosphere. As illustrated in Figure 3, the annular mode (EOF 1) characterizes over half of the variability of the zonally averaged 

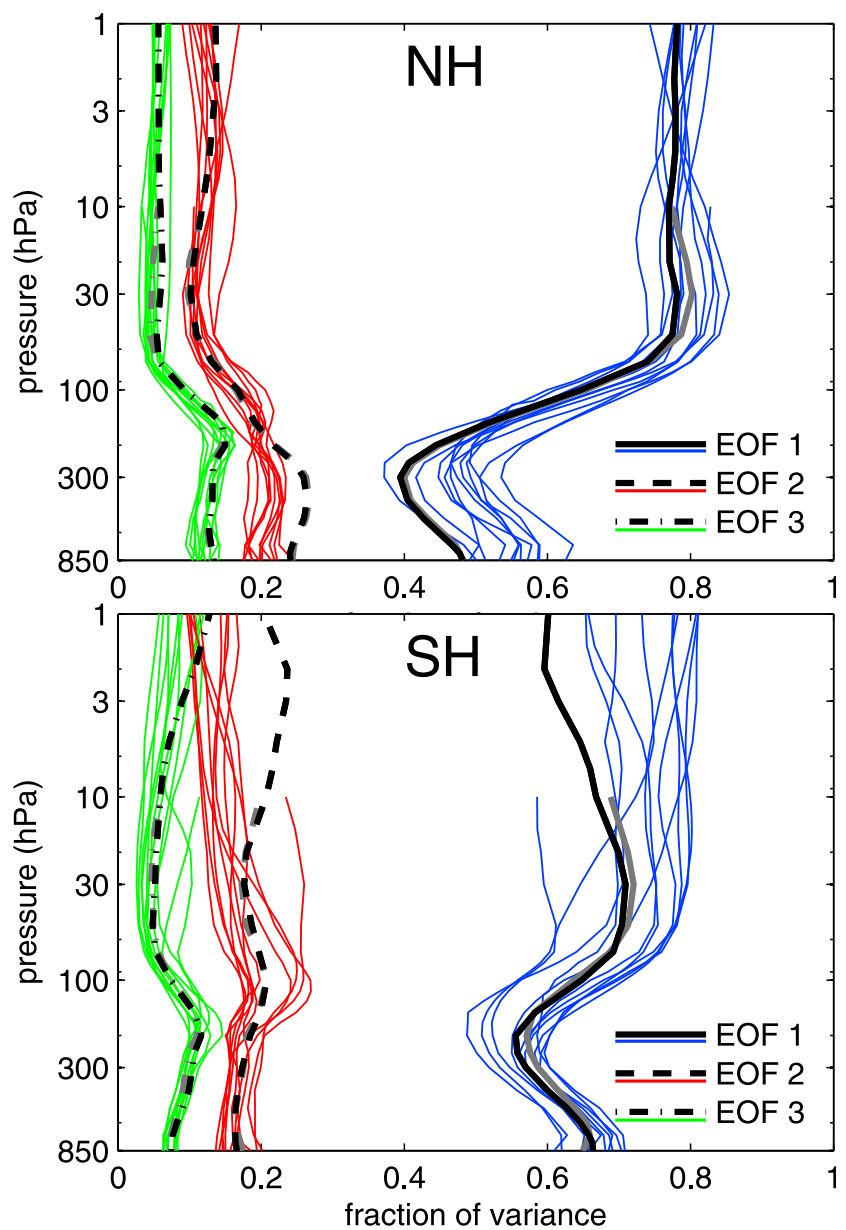

Figure 3. The fraction of variance of the zonal mean geopotential height characterized by the first three EOFs, as a function of pressure in the Northern and Southern hemispheres $(\mathrm{NH}, \mathrm{SH})$. Thick black and gray lines are based on ECMWF and NCEP-NCAR reanalyses, respectively, and thin colored lines show results from the models. The annular mode (EOF 1) captures over half the variance at most pressure levels in both hemispheres and dominates the variability of the stratosphere. Models simulate the structure of variability in the reanalyses quite well but tend to have some trouble capturing the asymmetry between the hemispheres; the annular mode characterizes too much of the variance in the $\mathrm{NH}$ troposphere and $\mathrm{SH}$ stratosphere.

flow at most levels in both hemispheres. In the NH stratosphere in particular, it characterizes over three quarters of the variance. The CCMs capture the same structure as in reanalyses but tend to overestimate the significance of the annular mode at the expense of variability in the second EOF in the NH troposphere and $\mathrm{SH}$ stratosphere. These biases suggest that models have some difficulty capturing the asymmetry between the two hemispheres, a theme that will appear in later diagnostics as well. Baldwin and Thompson [2009] show that the annular mode index is highly correlated with empirical measures such as the average geopotential height over the polar cap or strength of the zonal wind in the stratospheric vortex and tropospheric extratropical jets. For the purposes of comparing models with reanalyses, however, the annular mode provides a distinct advantage in that it adapts to biases in the climatology of models, particularly in the position of the tropospheric jets.

\subsection{Spatial Structure of the Annular Modes}

[20] We next assess the spatial structure of the model annular mode patterns. Figure 4 illustrates the NAM and SAM patterns for ERA-40 data, weighted by the cosine of latitude so that profiles reflect mass displacements. Note that each mode is computed from hemispheric anomalies alone; we illustrate their global structure by regressing the respective time series on the global zonal mean anomalies. By removing the global mean geopotential height at each time step, we guarantee that at each level the pattern reflects an equal and opposite displacement of mass between the polar cap and lower latitudes, even in the upper stratosphere. A qualitative change in the structure occurs near the tropopause: below $100 \mathrm{hPa}$, the pattern is confined mainly to one hemisphere. In the midlatitudes, the winds in geostrophic balance primarily reflect a meridional vacillation of the tropospheric eddy driven jet, and the node, or zero crossing, of the pattern is close to the latitude of the peak barotropic winds (not shown). Above $100 \mathrm{hPa}$, the equatorial lobe of the pattern elongates, stretching across the equator, and the winds in geostrophic balance (not shown) become more monopolar in character, primarily reflecting a variation
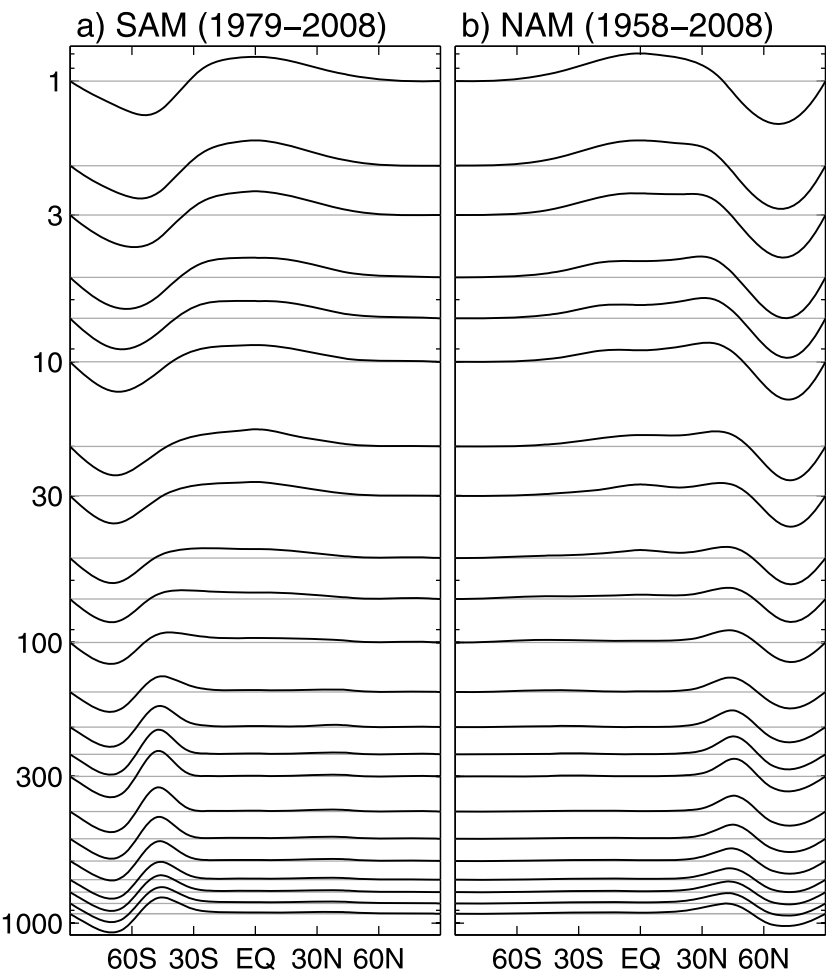

Figure 4. The SAM and NAM patterns, as a function of pressure, computed from ECMWF reanalyses for 19792008 and 1958-2008, respectively. Patterns have been weighted by the cosine of latitude, so that they reflect the mass balance. The NAM patterns and temporal structure (shown in Figures 5-11) based on data restricted to the satellite era are not significantly different from that based on the full record. 


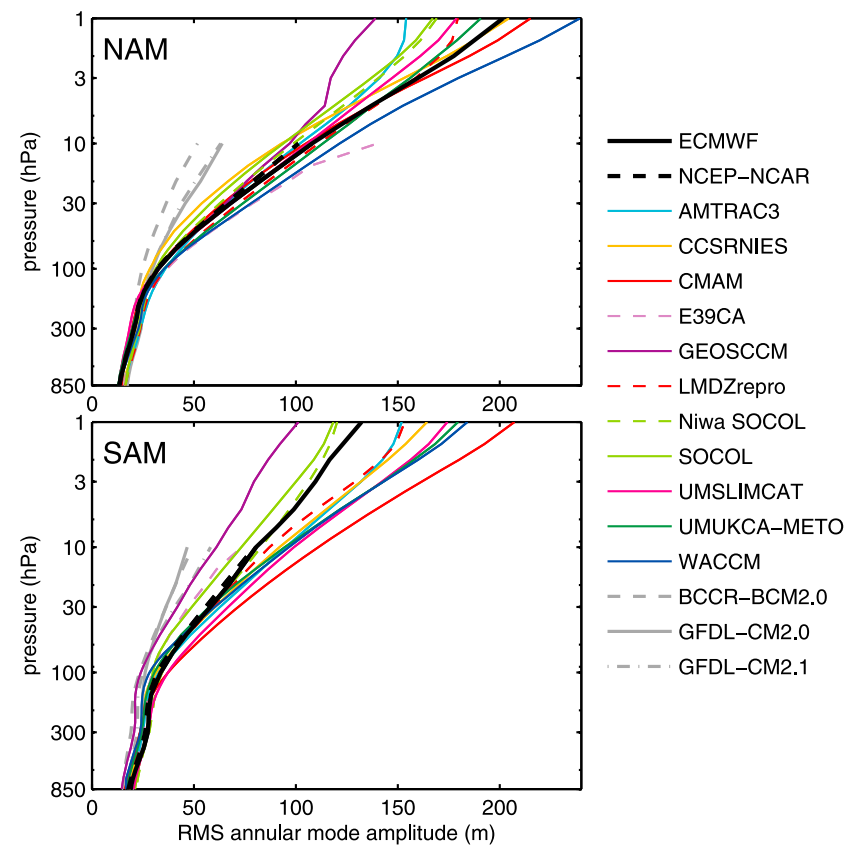

Figure 5. The root mean square amplitude of the annular mode pattern of variability in the CCMs as a function of pressure in the (top) NH and (bottom) SH. Analysis of the reanalyses and three IPCC-AR4 models are included for reference, and marked with black and gray lines, respectively.

in strength and size of the stratospheric polar vortex. As discussed in the context of Figure 1, climate trends have the largest impact on the annular mode near $100 \mathrm{hPa}$. Greenhouse gas forcing increases the tropopause height, so that levels that were once mostly in the stratosphere begin to develop a more tropospheric character.

[21] The generic dipole structure of the annular mode is well captured by all models, allowing us to characterize the spatial structure of their patterns with two variables, (1) the amplitude of mass displacement, and (2) the latitude of the node. Figure 5 illustrates the former, plotting the latitude weighted, root mean square amplitude of the annular mode pattern as a function of height, computed from reanalyses, the CCMVal-2 models, and three CMIP3 models for which stratospheric data is available. All of the CCMs capture the vertical structure of the annular mode through the depth of the atmosphere, albeit with increasing model spread in upper layers. In addition, we find that the CCMs simulate the amplitude of the annular modes in the stratosphere more accurately than the three CMIP3 models, particularly in the $\mathrm{NH}$. We note that the CMIP3 model GFDL-CM2.1 is related to the CCM AMTRAC3, differing by a coupled ocean versus chemistry and a well resolved stratosphere. In addition, nearly all CCMVal-2 models correctly capture the increased variance of the NAM in the stratosphere relative to the SAM. There is substantially more spread between the models and reanalyses in the $\mathrm{SH}$, suggesting less agreement between models in representing SH dynamics.

[22] Figure 6 characterizes the latitudinal structure of the annular mode, plotting the latitude of the node in the annular mode pattern as a function of pressure. The models capture the gross structure, with the equatorward shift in the node in the tropopause region, in step with the change in the character of the variability between the troposphere and stratosphere. As with the amplitude of the annular mode, the CMIP3 models appear to have more difficulty with the structure in the stratosphere, particularly in the $\mathrm{NH}$. There are also systematic biases: in the troposphere, the node is generally too far equatorward, reflecting a equatorward bias in the time mean eddy driven jets in both hemispheres. In the stratosphere, there is conversely a systematic poleward bias in the annular mode structure in the SH. There is also quite a spread in the sharpness of the transition between troposphere and stratosphere in the models. We must be cautious with the reanalyses here, however, as satellite retrievals may have difficulty resolving sharp vertical gradients in this region of the atmosphere. The combined effect of the biases makes the SAM looks more like the NAM in models, suggesting difficulty in simulating the asymmetry between hemispheres. Despite these deficiencies, however, the annular mode in the
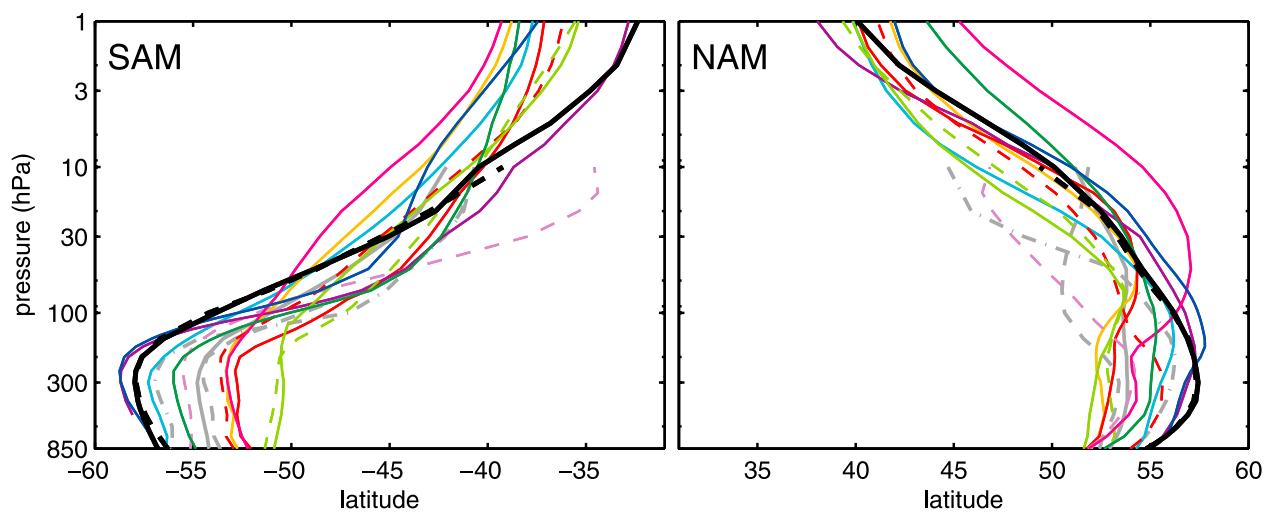

Figure 6. The latitude of the node of the annular mode patterns of variability as a function of pressure. Line colors correspond to the legend in Figure 5: thick black lines are based on ECMWF and NCEPNCAR reanalyses, thin-colored lines are based on the CCMs, and gray lines are based on the CMIP3 models. Note the significant latitude bias in the troposphere of both hemispheres, which reflects the fact that the tropospheric jet stream is too far equatorward in most models. 
a) NAM standard deviation, reanalysis

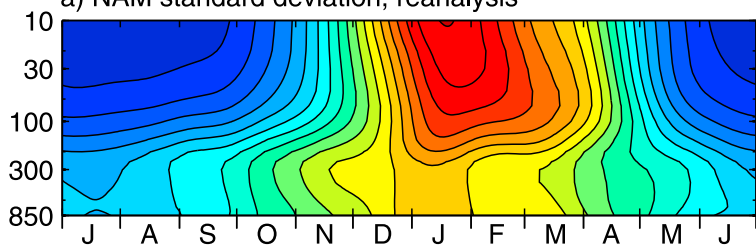

c) NAM standard deviation, REF-B1

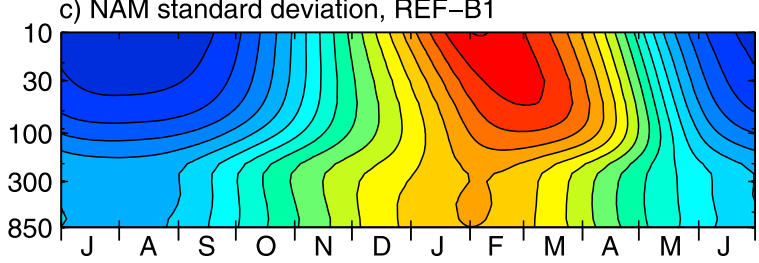

e) NAM standard deviation, REF-B2

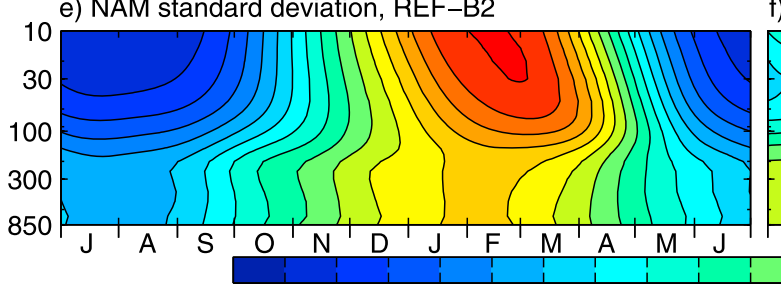

$\begin{array}{lllllllllllllllllllll}0.1 & 0.2 & 0.3 & 0.4 & 0.5 & 0.6 & 0.7 & 0.8 & 0.9 & 1 & 1.1 & 1.2 & 1.3 & 1.4 & 1.5 & 1.6 & 1.7 & 1.8 & 1.9 & 2\end{array}$ b) SAM standard deviation, reanalysis

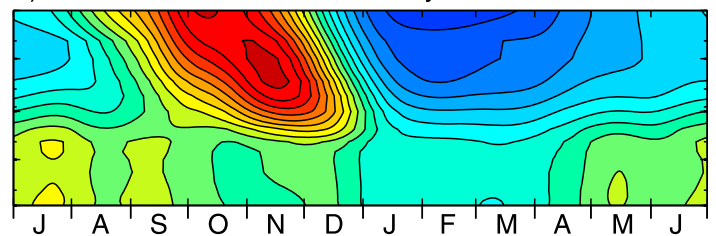

d) SAM standard deviation, REF-B1

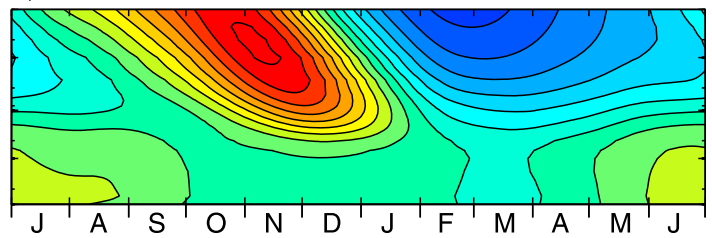

f) SAM standard deviation, REF-B2

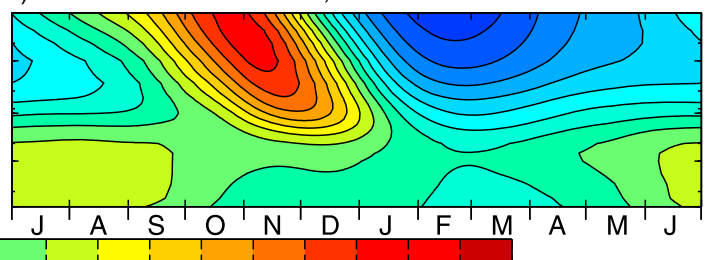

Figure 7. The standard deviation of the NAM and SAM indices as a function of season and pressure: $(a, b)$ the ECMWF reanalyses, (c, d) the multimodel ensemble mean for the late 20th century based on REF-B1 integrations, and (e, f) the multimodel ensemble mean for the late 21 st century based on the last 47 years of the REF-B2 integrations. Note that the indices have been normalized to have unit variance at all levels, removing the systematic increase in variance with height shown in Figure 5.

models clearly characterizes the same spatial variability as in the reanalyses. It is thus fair to compare the temporal structure of the models' annular mode directly with that in reanalyses.

\subsection{Temporal Structure of the Annular Modes}

[23] We show the seasonal and vertical structure of the annular mode variance in Figure 7 for ECMWF reanalyses, the multimodel mean from REF-B1 simulations of the late 20th century, and the multimodel mean from REF-B2 simulation of the late 21 st century. In computing the multimodel composite, we use all available ensemble integrations to compute the statistics for each individual model, but assign each model equal weight in the multimodel mean. For fair comparison, only models which provided REF-B2 integrations are included in the REF-B1 composite. Figure 7 is most appropriate for direct comparison, as here the models simulate the same period as the reanalyses, forced with observed SSTs. The models, both as a group and individually (not shown), simulate the structure quite well, capturing the marked asymmetry of the annular mode seasonal cycle between the two hemispheres. In the troposphere, the annular mode is most variable in the winter in both hemispheres, albeit with weaker seasonal variation in the $\mathrm{SH}$, a 6 month phase lag consistent with the annual variation in solar forcing. In contrast, the active periods of variability in the stratosphere are only two months out of phase with one another, peaking around November in the SH and January in the $\mathrm{NH}$.

[24] There are important differences between the models and reanalyses, however, which will become more clear in other metrics. We first focus on the timing of the seasonal cycle, particularly in the lower stratosphere. Variability of the vortex appears first in the upper stratosphere, penetrating lower in the stratosphere with time. The rate at which the variability descends, however, is too slow in the models. At $10 \mathrm{hPa}$, the seasonal cycle is about on time (perhaps even too early in the $\mathrm{SH}$ ), but just above the tropopause, the peak in variance is about a month late in both hemispheres and then lingers for too long. In the $\mathrm{NH}$, the vortex in the lower stratosphere is too steady in the early winter, and tends to remain variable too late; this is reflected in the general lack of Stratospheric Sudden Warmings (SSWs) in the early winter in many models, and a delay in the final warming [Eyring et al., 2010, Chapter 4]. In the SH, the prolonged variability in early January is associated with the delayed final breakdown of the vortex, as planetary wave propagation is cut off once the winds become easterly.

[25] Gerber et al. [2008a] found that the temporal variability of the annular mode in the troposphere was relatively unchanged between the 20th and 21st centuries in CMIP3 integrations. The CCMs provide predictions for an extended vertical domain. The bottom panels of Figure 7 illustrate the annular mode variance structure based on statistics from the last 47 years of the 21 st century in the REF-B2 runs. We choose this length of analysis for the most fair comparison with the REF-B1 integrations. There is remarkably little change in the variance throughout the depth of the atmosphere in both hemispheres, with the exception of the $\mathrm{SH}$ stratosphere. Here we find a slight weakening in the amplitude of the seasonal cycle and shift in the active period earlier in the seasonal cycle. The shift in the ensemble mean 
a) NAM time scale, reanalysis (days)

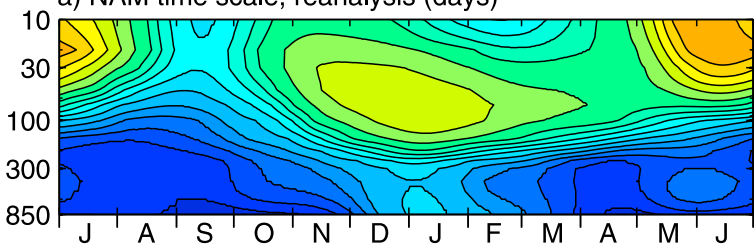

c) NAM time scale, REF-B1 (days)

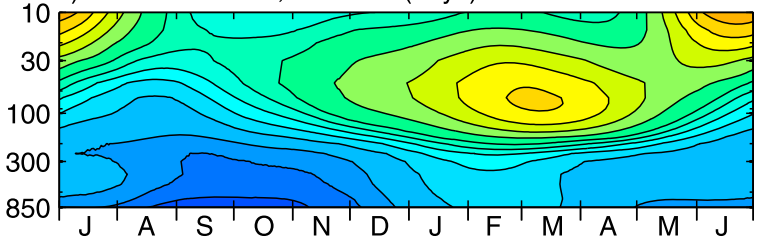

e) NAM time scale, REF-B2 (days)

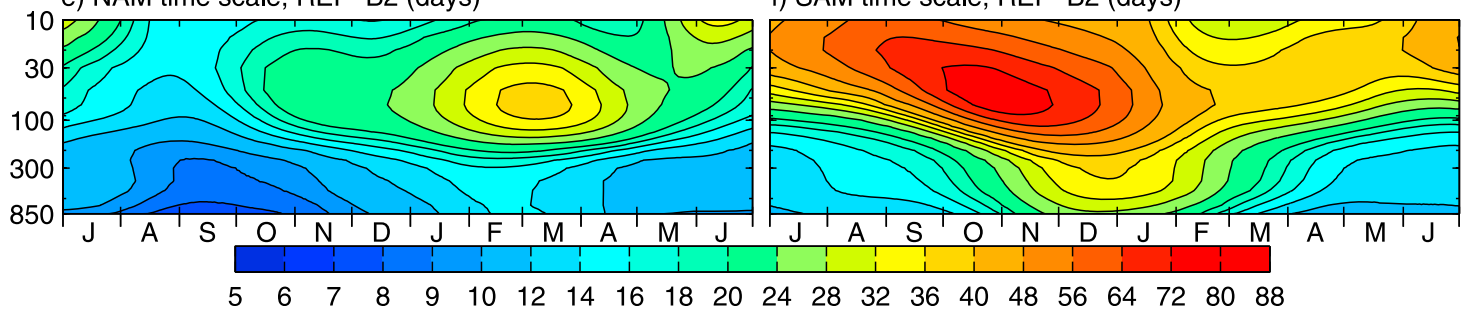

b) SAM time scale, reanalysis (days)

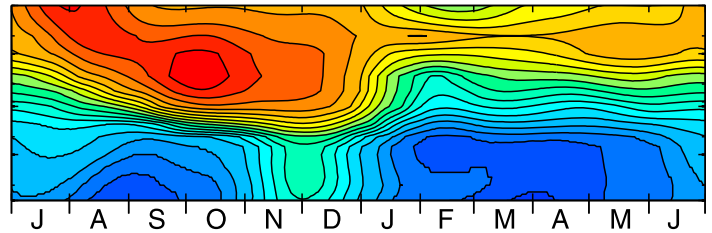

d) SAM time scale, REF-B1 (days)

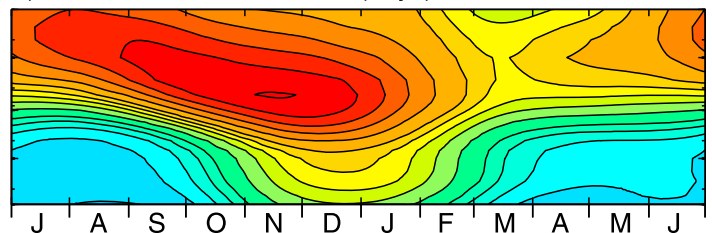

f) SAM time scale, REF-B2 (days)

Figure 8. The same as Figure 7, but here the e-folding time scale of the NAM and SAM indices as a function of season and pressure.

is only marginally significant when compared against intermodel differences, but recovery of the ozone hole is expected to accelerate the final transition to easterlies [Eyring et al., 2010, Chapter 4], thereby closing off wave induced variability earlier in the season. The weakening of the peak variance suggests less variation in the date of the final warming after ozone recovery. The lack of change in the variance in the $\mathrm{NH}$ suggest that its polar vortex remains equally variable for the rest of the 21 st century, consistent with the findings of McLandress and Shepherd [2009] for a single CCM. Despite the consistency of the internal variability, however, they found that the frequency of SSWs can exhibit trends due to changes in the mean state, because SSWs are defined by a fixed threshold, the reversal of the zonal mean winds at $60^{\circ} \mathrm{N}$ and $10 \mathrm{hPa}$. This highlights the need to carefully define metrics of internal variability so that they are not affected by trends in the climatology.

[26] Baldwin et al. [2003] quantified the persistence of annular mode variability as a function of season and height with the e-folding time scale of its autocorrelation function. The time scale provides an integrated measure of variability on all time scales, but to first-order provides a rough estimate of the persistence annular mode anomalies. Figure 8 illustrates the annular mode time scales for ECMWF reanalyses and CCMs. We first note that it is important to interpret the time scales in the context of the variance structure shown in Figure 7. The time scales are most meaningful when the annular mode is active; the extreme persistence in the $\mathrm{NH}$ summer above $30 \mathrm{hPa}$, for instance, occurs during a period of stratospheric easterlies when there is almost no variability of the NAM, and could be influenced by small variations in total column ozone left over from the previous winter [Fioletov and Shepherd, 2003]. Second, we note that the time scale represents a seasonally localized decay rate of anomalies. The long time scales in the SH stratosphere in winter and spring indicate that anomalies in of the SAM decay very slowly during these seasons; one can infer that if the vortex is weak or strong in midwinter, is likely to remain so right up until the final warming. The drop in persistence after the final warming, however, suggests that little memory of the winter vortex is retained after the transition to easterlies. The time scale estimated in October, however, will not reflect this break as it measures the persistence of anomalies in a seasonally local window around October.

[27] We find that the models simulate the NH-SH asymmetry in the seasonal cycle of the annular mode e-folding time scale, both in the troposphere and stratosphere, and the tendency toward longer time scales in the SH. In addition, the models' time scales appear to be quite stable in time; the multimodel ensemble mean is not statistically different at the end of the 21 st century as compared to the end of the 20th. There are, however, significant differences between the reanalysis and model time scales. In the troposphere, the CCM biases are similar to those of the CMIP 3 models [Gerber et al., 2008a]: the models overestimate the annular mode time scales, particular in the SH (note the nonlinearity of the color scale), and the seasonal cycle is delayed and broader. In the stratosphere, the time scales are of the right magnitude, but the seasonal evolution is delayed, particularly in the $\mathrm{NH}$.

[28] We have shown that models accurately simulate the annular mode spatial patterns, and the seasonal evolution of the variance in the troposphere, phenomena likely controlled primarily by tropospheric dynamics [Vallis and Gerber, 2008]. They have more trouble, however, getting the seasonal structure of the persistence, exhibiting a delay in both hemispheres that is more consistent with biases in the stratosphere than in the troposphere. While the model biases 
a) NAM predictability, reanalysis

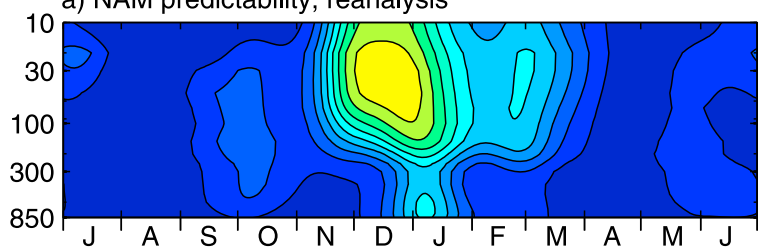

c) NAM predictability, REF-B1

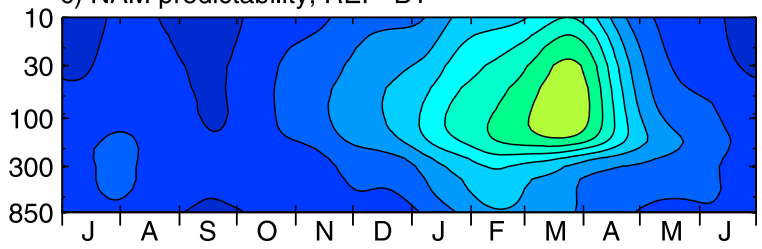

e) NAM predictability, REF-B2

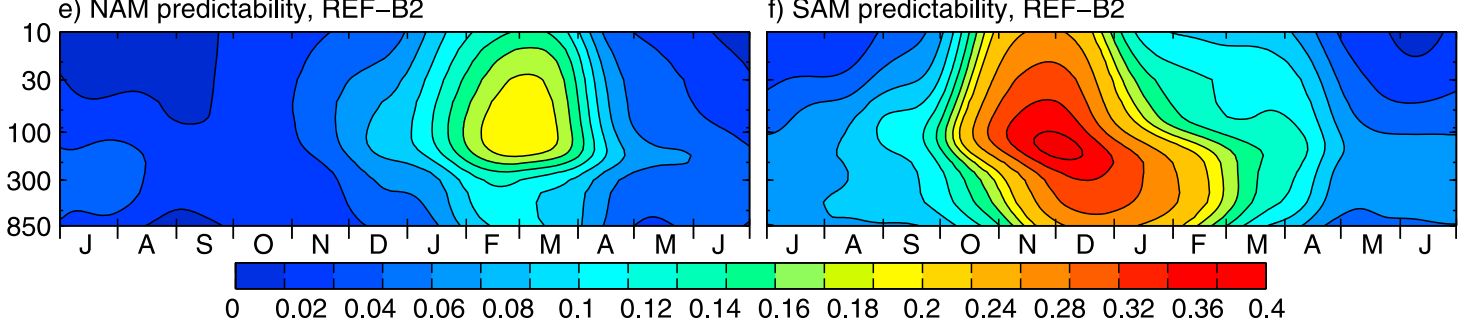

b) SAM predictability, reanalysis

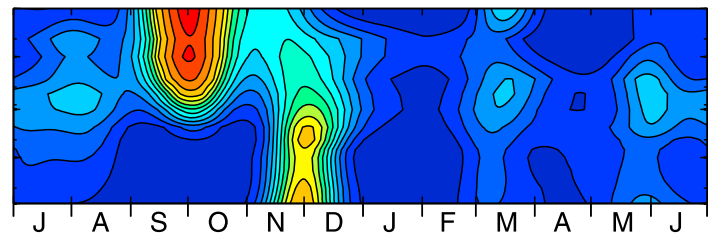

d) SAM predictability, REF-B1

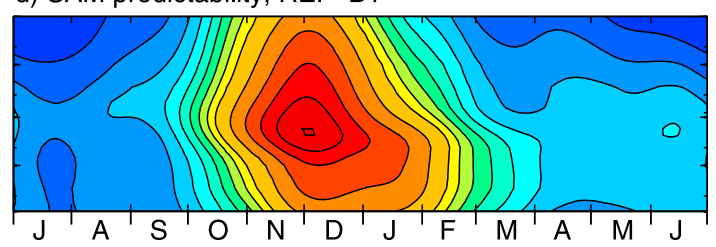

f) SAM predictability, REF-B2

Figure 9. The same as Figure 7, but here the fraction of the variance of the 30 day mean $850 \mathrm{hPa}$ annular mode index, lagged by 10 days, that is linearly correlated with the instantaneous annular mode index as a function of season and pressure.

reveal weaknesses of the models, they may actually help elucidate the influence of the stratosphere on the troposphere. In both reanalyses and the models, increased annular mode persistence in the troposphere is associated with increased variance of the annular mode in the lower stratosphere. The time scales of the variability in the stratosphere are generally longer than those in the troposphere, but impact the troposphere when variations in the lower stratosphere are large enough to influence tropospheric circulation [Gerber and Polvani, 2009].

[29] To cement the connection between biases in the lower stratosphere and troposphere in the models, we repeat the analysis of Baldwin et al. [2003, Figure 2]. Figure 9 plots, as a function of height and season, the fraction of the variance of the 30 day mean $850 \mathrm{hPa}$ annular mode index, lagged by 10 days, that can be "predicted" from a persistence forecast based on today's instantaneous annular mode index. This quantity is the square of the correlation between the annular mode index on a particular day and level, and the 30 day mean annular mode index after a 10 day lead (i.e., 10-40 days ahead). The statistic confirms the surprising result that, at some times of the year, the stratosphere is a better predictor for the near surface annular mode index than information about the near surface annular mode itself. We first focus on the NH. In the reanalyses, the stratospheric NAM index during December can be used to predict over $20 \%$ of the variance in the monthly mean January index at the surface. In the troposphere, a similar persistence forecast explains only about half as much of the variance. The models exhibit similar behavior, but the connection is delayed, starting about a month too late, and peaking almost 3 months later than in the reanalyses.
[30] The source of the increased predictability in the stratosphere is related to the downward propagation of NAM anomalies [Baldwin and Dunkerton, 2001; Christiansen, 2005] and the increased memory of the stratospheric flow [Gerber et al., 2009]. This behavior is well captured by both comprehensive and idealized GCMs [e.g., Christiansen, 2001; Gerber and Polvani, 2009, respectively], and the CCMs and CMIP3 models simulate the propagation fairly well, as shown in Figure 10. The tropospheric response to the stratospheric vortex is quite weak in some models, and not statistically significant for any given integration, but consideration of all models together yields a significant, albeit weak impact. Figure 9, however, suggests that the timing and impact of the coupling differs significantly between reanalyses and models. In reanalyses, the increased predictability is associated with early and midwinter variability of the stratospheric vortex, and has largest impact in January, when the surface annular mode is most persistent. In the models, however, it appears that much of the signal is associated with the final warming of the stratospheric vortex, and reflects the delay in the period of increased persistence of NAM anomalies in both the stratosphere and troposphere seen in Figure 8.

[31] The predictability relationship in the Southern Hemisphere is less clear. With the reanalyses, we must restrict the sampling period to the satellite era, so that the correlation is less statistically certain. With the models, the bias toward very long time scales leads to spurious predictability, but there is evidence of delayed and downward coupling between the lower stratosphere and the near surface, likely associated with the final warming of the vortex. In a more detailed study of the GEOSCCM model, Fogt et al. [2009] find that models can overpredict the relationship between spring ozone and 

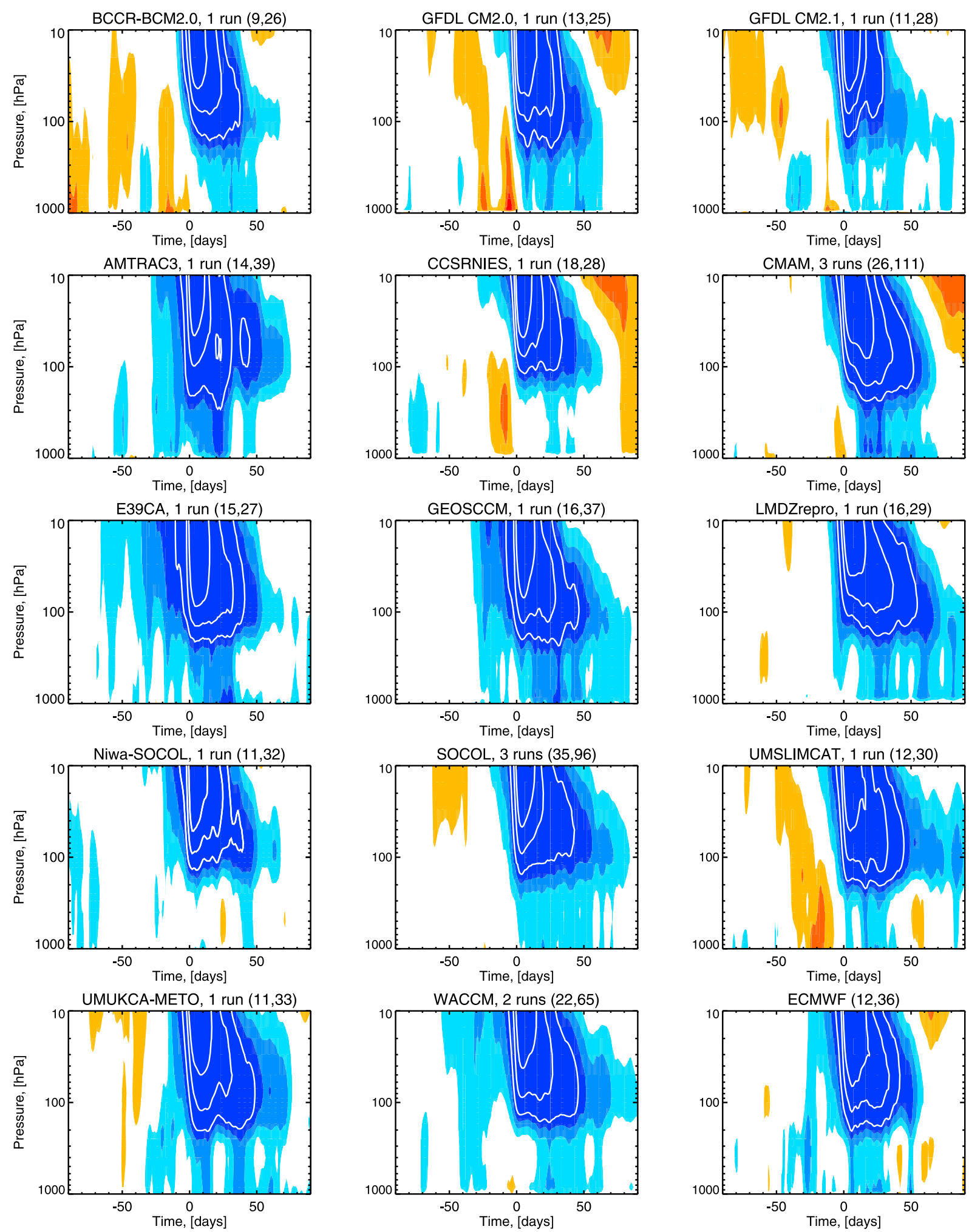

Figure 10. Composite differences of the NAM index between strong and weak stratospheric events. Results are shown for the three CMIP3 models (top row), 11 CCMVal-2 model REF-B1 simulations, and ECMWF reanlaysis. Day 0 corresponds to the onset of the stratospheric event at $10 \mathrm{hPa}$, defined by the time the NAM index crosses a \pm 2 standard deviation threshold. The shading interval is 0.5 standard deviations (beginning at \pm 0.5 ), with contours at 1 standard deviation intervals. Blue shading denotes positive values in the NAM index. The numbers above each frame indicate the number of strong and weak events included in the composite. Given the persistence of stratospheric events, a maximum of one event is considered for any given winter. 

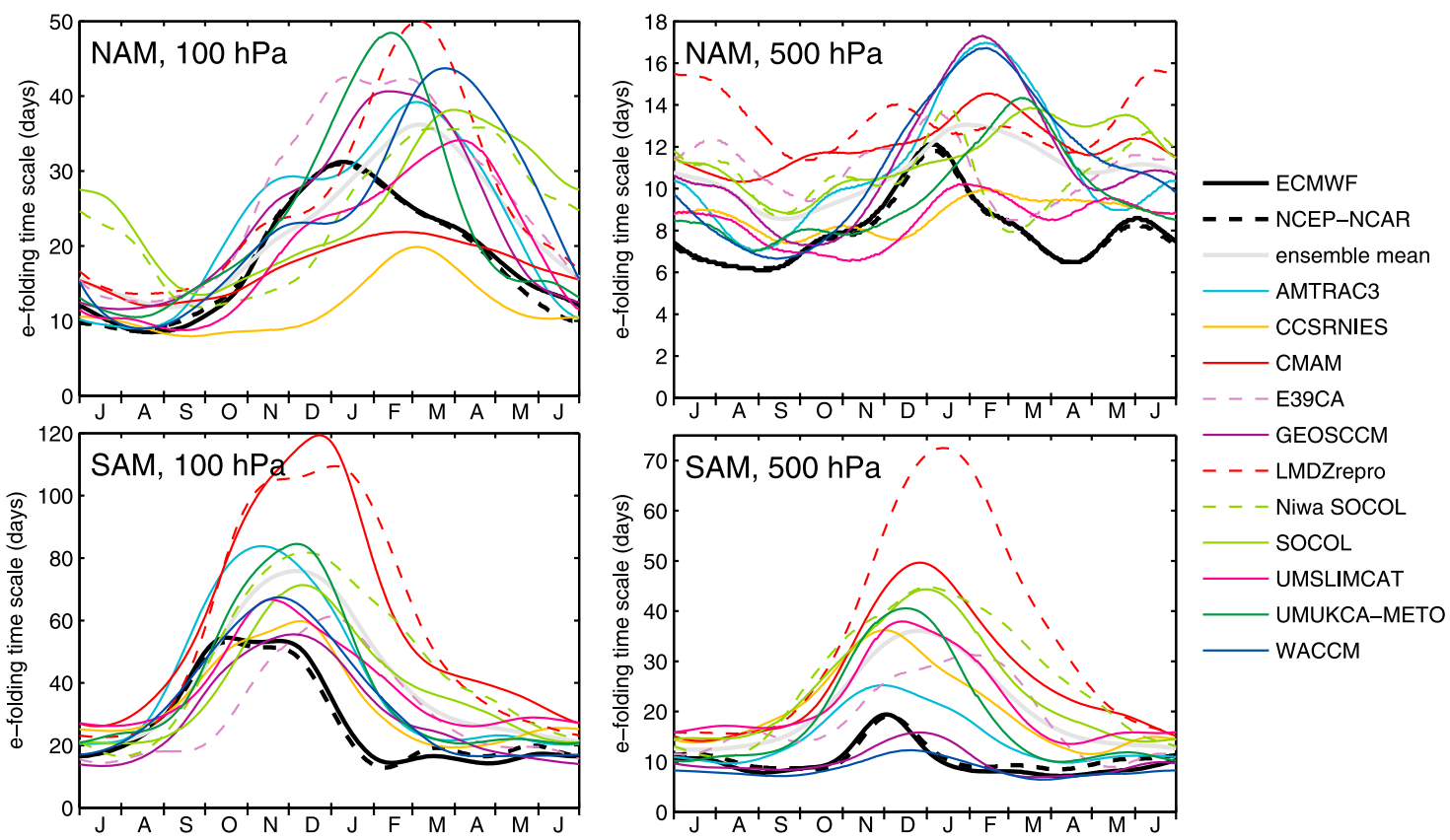

Figure 11. The annular mode e-folding time scale in (left) the lower stratosphere and (right) the midtroposphere as a function of season for the individual CCMs: (top) NH and (bottom) SH.

austral summer SAM as a consequence of the delayed breakdown of the polar vortex. The trend in the multimodel composite to the end of the 21 st century is a slight shift of the maximum predictability to early times in the year, consistent with an earlier final warming due to recovery of the ozone hole.

\subsection{Assessment of Individual Models}

[32] The analyses in Figures 7-9 applied to individual models (not shown) indicates that each captures the key elements of the temporal structure, but there is significant spread, particularly in the annular mode time scales and predictability metrics. The annular mode e-folding time scales of each model at 100 and $500 \mathrm{hPa}$ are shown in Figure 11. Given the stability of model time scales, we use all available ensemble members of the REF-B0, B1, and B2 integrations to increase the statistical confidence. Most models capture the gross features of the seasonal cycle of variability in both hemispheres. The seasonal cycle of the tropospheric NAM, and evidence of downward coupling, however, is not always robust. In addition, the delay in the seasonal cycle and overestimation of time scales shown in the multimodel ensemble mean in Figure 8 is a common bias. Differences between the models are most extreme in the Southern Hemisphere. The SAM time scales vary by a factor of 2 in the stratosphere and by a factor of 4 in the troposphere. The overestimation of stratospheric time scales in the $\mathrm{NH}$, while not as extreme, can be seen in NAM composites of Figure 10.

[33] The GEOSCCM and WACCM, and, to a lesser extent AMTRAC3, models quite accurately match the observations in the $\mathrm{SH}$ troposphere, skill not achieved by the CMIP3 models analyzed in the work of Gerber et al. [2008a, Figure 2]. It is worthy of note that these models also have the most accurate spatial structure, illustrated in
Figure 6; the latitude of the node of the annular mode reflects the climatological position of the jet. Kidston and Gerber [2010] found that accurate simulation of the climatology in the CMIP3 models also increased the fidelity of their internal variability, and this link in the CCMs is further explored by Son et al. [2010]. It is also interesting that these three models with the shortest time scales in the $\mathrm{SH}$ exhibit the longest time scales in the $\mathrm{NH}$ : each has a peak in persistence in JFM that is significantly higher than that observed. This may indicate that model biases in the SAM are unrelated to biases in the NAM. Closer inspection shows that the NAM time scales in these models have a very strong seasonal cycle, and that their time scales are among the shortest in other seasons, when there is no active coupling between the troposphere and stratosphere. This suggests that the extended persistence of the NAM in winter could be associated with artificially strong coupling with the stratosphere in these models.

\section{Discussion}

[34] Keeley et al. [2009] emphasize that the e-folding time scale provides an integrated measure of variability on all time scales, and can be strongly influenced by variability on interannual time scales. As shown by Baldwin and Dunkerton [2001], a single Stratospheric Sudden Warming (SSW) event can lead to anomalies on 2-3 month time scales. While the dynamics of such events are more intraseasonal in character, particularly their swift onset, their impact will strongly influence the seasonal mean signal, and so could be interpreted as interannual variability. Particular care, then, must be taken in interpreting the annular mode e-folding time scales and the influence of the stratosphere. From a dynamical perspective, one could argue that SSWs (or final warmings in the $\mathrm{SH}$ ) are a source of intraseasonal variability of the 


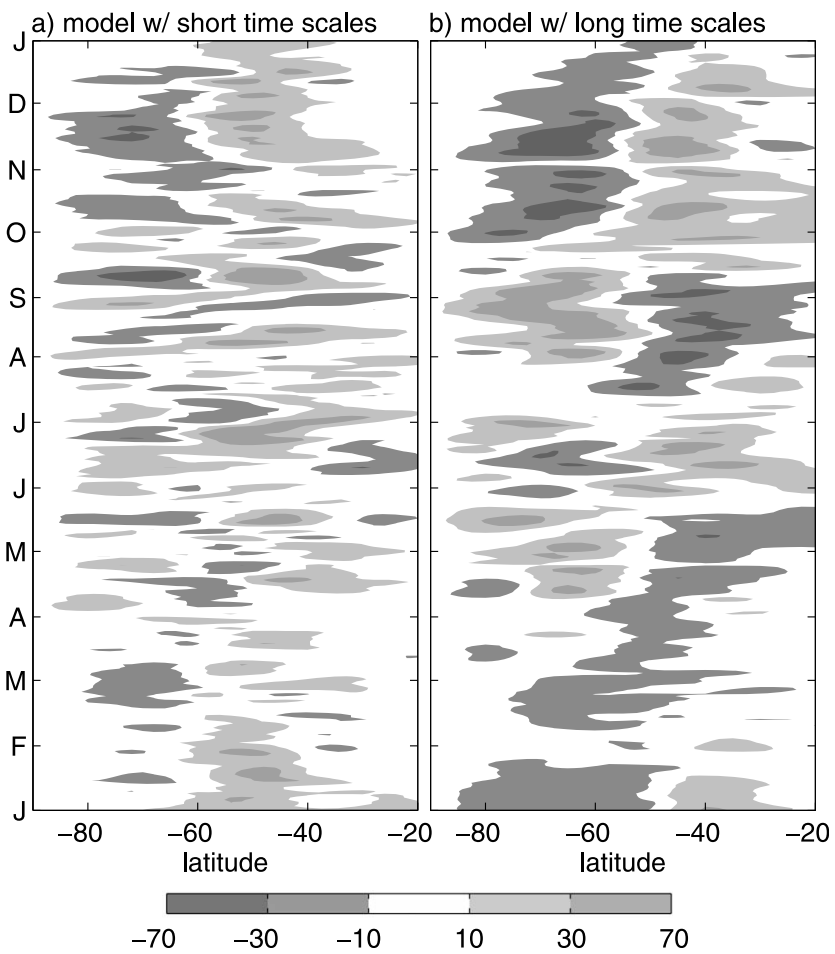

Figure 12. Hövmöller diagrams based on one year of $\mathrm{SH}$ height anomalies at $500 \mathrm{hPa}$ for (a) GEOSCCM, a model with short SAM time scales, and (b) LMDZrepro, a model with long time scales. A slowly varying seasonal climatology has been removed from $Z$, but otherwise the data are unfiltered. Even in the raw height fields, one case sees quantitative differences in the variability of the two models, which are reflected in the time scales shown in Figure 11.

annular mode. On the other hand, for a given winter, there is generally either an SSW or not, and the interannual variation in events could be viewed as an external signal, as in the work of Keeley et al. [2009].

[35] Given the complexity of the time scales, it is instructive to return to the raw data. In Figure 12 we compare one randomly selected year of SH geopotential height anomalies at $500 \mathrm{hPa}$ for two models near the extrema of the multimodel data set in terms of the SAM time scales shown in Figure 11. The slowly varying seasonal climatology has been removed, but the data is otherwise unfiltered. Here one can see a quantitative difference in the variability. Geopotential height anomalies in the model on the left are clearly less persistent than anomalies in the model shown on the right. Anomalies in the reanalyses (not shown) are comparable to those in Figure 12a, as this model has comparable SAM time scales. Note also that both panels hint at the increased persistence in NDJ, though we should be cautious of over interpreting a single year. The time scales shown in Figure 11 reflect statistics based on the entire data set, confirming that there is greater persistence during this period. The differences in structure shown in Figure 12 are not sensitive to the particular choice of models shown here. Similar differences are found between any two models with different annular mode time scales.

\section{Conclusions}

[36] We have evaluated the internal variability simulated by chemistry-climate models contributed to the CCMVal-2 modeling activity by comparing their annular mode variability with that in reanalyses. The annular modes provide an ideal metric to assess the coupling between stratospheric polar vortex and tropospheric midlatitude jets. After refining the Baldwin and Thompson [2009] procedure for computing the annular modes to ensure that they are robust to secular trends in long data sets, we found that the internal variability and vertical coupling of the atmosphere is remarkably consistent in model integrations of the entire 21 st century, despite large changes in the climatology at some levels. Using the metrics of Baldwin and Dunkerton [2001] and Baldwin et al. [2003], we find that the models capture the key features of observed intraseasonal variability though the depth of the atmosphere, including (1) the marked asymmetry in the seasonal cycle between the Northern and Southern hemispheres, (2) the strong gradients in the character of variability with height, and (3) the coupling between stratosphere and troposphere during the active seasons, winter in the $\mathrm{NH}$ and spring-summer in the $\mathrm{SH}$.

[37] There is, however, significant spread between the $\mathrm{CCMs}$, and several common biases. In the troposphere, the annular mode pattern in models is generally shifted equatorward and too persistent, particularly in the SH summer, a bias similar to that found in CMIP3 coupled climate models [Gerber et al., 2008a]. In some models the bias in the SH is extreme, with time scales up to four times those observed, but two CCMs appear to capture the variability quite well, better than all CMIP3 models. The better performing models tend to have higher horizontal resolution, which may allow them to better simulate eddy-mean flow interactions [Gerber et al., 2008b]. In the stratosphere, the seasonal evolution of variability in the models is well captured, but almost uniformly delayed in both hemispheres, particularly at lower levels; the delay can be seen both in the variance and time scale of the annular modes.

[38] The consistency of the delay in the seasonal cycle suggests active coupling between the troposphere and stratosphere. Comparison between Figures 7 and 8 indicates a close relationship between increased variance of the annular mode in the lower stratosphere and increased persistence of the annular mode in the troposphere in both the reanalyses and models. One does not find this connection between variance and persistence in the troposphere; for example, greater variance in the troposphere during the austral winter does not correlate with longer persistence. As suggested by Gerber and Polvani [2009], the tropospheric annular mode becomes more persistent when there is stronger variability in the stratosphere; at these times, the longer time scales of the lower stratosphere can impact tropospheric persistence. The delayed coupling is most evident in the predictability analysis shown in Figure 9. In the NH, models suggest coupling in the late winter and early spring associated with late SSWs and the final warming, while the strongest connection in observations is associated with SSWs in the early and mid winter. In the $\mathrm{SH}$, the coupling is associated with the final warming in both reanalyses and models, but the transition occurs too late in models. With the recovery of the ozone 
hole, however, the coupling shifts earlier in the season. The connection between model biases in the troposphere and stratosphere also suggests a coupled origin of the model deficiencies. Stratospheric variability is primarily driven by upward propagating planetary waves from the troposphere, and so depends on both the tropospheric representation of planetary waves and the propagation and breaking of waves within the stratosphere itself. The tropospheric response to stratosphere, in turn, likely depends on details of the variability in the lower stratosphere and eddy-mean flow interactions in the troposphere [e.g., Gerber et al., 2009].

[39] The annular mode has a first-order impact on regional climate and weather [e.g., Thompson and Wallace, 2001; Baldwin and Dunkerton, 2001], and so proper simulation of internal variability is important in and of itself for regional climate prediction. The difference in the persistence of jet anomalies shown in Figure 12 will have significant impacts on the seasonal variance on a regional level. There is also growing evidence that internal atmospheric variability can couple to the global climate, modulating carbon dioxide uptake in the Southern Ocean [Lovenduski et al., 2007] and influencing glacial melt rates by modifying warm water transport in the ocean [Holland et al., 2008]. It is thus important that the biases in internal variability be accounted for in analyses based on CCMVal-2 model output. Lastly, the overestimation of the annular mode time scales may influence the sensitivity of the models to external forcing [Gerber et al., 2008b; Ring and Plumb, 2008]. In particular, the tropospheric jets in models with long time scales may be more sensitive to external forcing [Gerber et al., 2008a; Kidston and Gerber, 2010; Son et al., manuscript in preparation, 2010].

[40] Accurate simulation of internal variability will likely become more important in the future as models begin to incorporate more climate feedbacks. To this end, it is noteworthy that capturing the temporal variability in one hemisphere or season does not necessarily mean capturing it in another. The CCMs with the shortest (and so most realistic) SAM time scales also exhibit the shortest NAM time scales in the boreal summer and fall, but the longest NAM time scales in the winter and spring. This suggests a perhaps excessive influence of the stratosphere on the troposphere, and, along with the link between model climatology and internal variability, indicates that there are still open questions in the modeling of large-scale dynamics.

[41] Acknowledgments. We thank Judith Perlwitz and two anonymous reviewers for constructive comments on earlier manuscript. E.P.G. acknowledges support from the National Science Foundation (NSF) under the Atmospheric and Geospace Sciences program. M.P.B. was funded by the NSF under the US CLIVAR program and the Office of Polar Programs. N.B. and S.H. were supported by the Joint DECC and Defra Integrated Climate Programme-DECC/Defra (GA01101). We acknowledge the modeling groups for making their simulations available for this analysis, the CCMVal Activity for WCRP's SPARC (Stratospheric Processes and their Role in Climate) project for organizing and coordinating the CCM data analysis activity, the British Atmospheric Data Center (BADC) for collecting and archiving the CCMVal model output, and the Program for Climate Model Diagnosis and Intercomparison (PCMDI) and the WCRP's Working Group on Coupled Modeling (WGCM) for their roles in making available the WCRP CMIP3 multimodel data set. Support for the CMIP3 data set is provided by the Office of Science, U. S. Department of Energy. CCSRNIES research was supported by the Global Environmental Research Fund of the Ministry of the Environment of Japan (A-071), and simulations were completed with the super computer at CGER, NIES.

\section{References}

Baldwin, M. P., and T. J. Dunkerton (1999), Propagation of the Arctic Oscillation from the stratosphere to the troposphere, J. Geophys. Res. 104(D24), 30,937-30,946, doi:10.1029/1999JD900445.

Baldwin, M. P., and T. J. Dunkerton (2001), Stratospheric harbingers of anomalous weather regimes, Science, 294, 581-584.

Baldwin, M. P., and D. W. J. Thompson (2009), A critical comparison of stratosphere-troposphere coupling indices, Q. J. R. Meteorol. Soc., 135, 1661-1672.

Baldwin, M. P., D. B. Stephenson, D. W. J. Thompson, T. J. Dunkerton, A. J. Charlton, and A. O'Neill (2003), Stratospheric memory and skill of extended-range weather forecasts, Science, 301, 636-640.

Benedict, J. J., S. Lee, and S. B. Feldstein (2004), Synoptic view of the North Atlantic Oscillation, J. Atmos. Sci., 61, 121-144.

Christiansen, B. (2001), Downward propagation of zonal mean zonal wind anomalies from the stratosphere to the troposphere: Models and reanalyses, J. Geophys. Res., 106(D21), 27,307-27,322, doi:10.1029/ 2000JD000214.

Christiansen, B. (2005), Downward propagation and statistical forecast of the near-surface weather, J. Geophys. Res., 110, D14104, doi:10.1029/ 2004JD005431.

Eyring, V., et al. (2005), A strategy for process-oriented validation of coupled chemistry-climate models, Bull. Am. Meteorol. Soc., 86, 1117-1133, doi:10.1175/BAMS-86-8-1117.

Eyring, V., et al. (2008), Overview of the new CCMVal reference and sensitivity simulations in support of upcoming ozone and climate assessments and the planned SPARC CCMVal, SPARC Newsl., 30, 20-26.

Eyring, V., T. G. Shepherd, and D. W. Waugh (Eds.) (2010), SPARC Report on Chemistry Climate Model Validation, SPARC Rep. 5, WCRP-X. (Available at http://www.atmosp.physics.utoronto.ca/SPARC)

Fioletov, V. E., and T. G. Shepherd (2003), Seasonal persistence of midlatitude total ozone anomalies, Geophys. Res. Lett., 30(7), 1417, doi:10.1029/2002GL016739.

Fogt, R. L., J. Perlwitz, S. Pawson, and M. A. Olsen (2009), Intra-annual relationships between polar ozone and the SAM, Geophys. Res. Lett., 36, L04707, doi:10.1029/2008GL036627.

Gerber, E. P., and L. M. Polvani (2009), Stratosphere-troposphere coupling in a relatively simple AGCM: The importance of stratospheric variability, J. Clim., 22, 1920-1933.

Gerber, E. P., and G. K. Vallis (2005), A stochastic model for the spatial structure of annular patterns of variability and the NAO, J. Clim., 18, $2102-2118$

Gerber, E. P., and G. K. Vallis (2007), Eddy-zonal flow interactions and the persistence of the zonal index, J. Atmos. Sci., 64, 3296-3311.

Gerber, E. P., L. M. Polvani, and D. Ancukiewicz (2008a), Annular mode time scales in the Integovernmental Panel on Climate Change Fourth Assessment Report models, Geophys. Res. Lett., 35, L22707, doi:10.1029/2008GL035712.

Gerber, E. P., S. Voronin, and L. M. Polvani (2008b), Testing the annular mode autocorrelation timescale in simple atmospheric general circulation models, Mon. Weather Rev., 136, 1523-1536.

Gerber, E. P., C. Orbe, and L. M. Polvani (2009), Stratospheric influence on the tropospheric circulation revealed by idealized ensemble forecasts, Geophys. Res. Lett., 36, L24801, doi:10.1029/2009GL040913.

Graversen, R. G., and B. Christiansen (2003), Downward propagation from the stratosphere to the troposphere: A comparison of the two hemispheres, J. Geophys. Res., 108(D24), 4780, doi:10.1029/2003JD004077.

Holland, D. M., R. H. Thomas, B. deYoung, and M. H. Ribergaard (2008), Acceleration of Jakobshavn Isbrae triggered by warm subsurface ocean waters, Nat. Geosci., 1, 659-664, doi:10.1038/ngeo316.

Kalnay, E., et al. (1996), The NCEP/NCAR 40 year reanalysis project, Bull. Am. Meteorol. Soc., 77, 437-471.

Keeley, S. P. E., R. T. Sutton, and L. C. Shaffrey (2009), Does the North Atlantic Oscillation show unusual persistence on intraseasonal time scales?, Geophys. Res. Lett., 36, L22706, doi:10.1029/2009GL040367.

Kidston, J., and E. P. Gerber (2010), Intermodel variability of the poleward shift of the austral jet stream in the CMIP3 integrations linked to biases in 20th century climatology, Geophys. Res. Lett., 37, L09708, doi:10.1029/ 2010 GL042873.

Lorenz, D. J., and D. L. Hartmann (2001), Eddy-zonal flow feedback in the Southern Hemisphere, J. Atmos. Sci., 58, 3312-3327.

Lovenduski, N. S., N. Gruber, S. C. Doney, and I. D. Lima (2007), Enhanced $\mathrm{CO} 2$ outgassing in the Southern Ocean from a positive phase of the Southern Annular Mode, Global Biogeochem. Cycles, 21, GB2026, doi:10.1029/2006GB002900.

Lu, H., L. J. Gray, M. P. Baldwin, and M. J. Jarvis (2009), Life cycle of the QBO-modulated 11 year solar cycle signals in the Northern Hemispheric winter, Q. J. R. Meteorol. Soc., 135, 1030-1043. 
McLandress, C., and T. G. Shepherd (2009), Impact of climate change on stratospheric sudden warmings as simulated by the Canadian Middle Atmosphere Model, J. Clim., 22, 5449-5463, doi:10.1175/2009JCLI3069.1.

Meehl, G. A., C. Covey, T. Delworth, M. Latif, B. McAvaney, J. F. B Mitchell, R. J. Stouffer, and K. E. Taylor (2007), The WCRP CMIP3 multimodel data set, Bull. Am. Meteorol. Soc., 88, 1383-1394.

Morgenstern, O., et al. (2010a), Anthropogenic forcing of the Northern Annular Mode in CCMVal-2 models, J. Geophys. Res., doi:10.1029/ 2009JD013347, in press.

Morgenstern, O., et al.. (2010b), Review of the formulation of presentgeneration stratospheric chemistry-climate models and associated external forcings, J. Geophys. Res., doi:10.1029/2009JD013728, in press.

North, G. R., T. L. Bell, R. F. Cahalan, and F. J. Moeng (1982), Sampling errors in the estimation of empirical orthogonal functions, Mon. Weather Rev., 110, 699-706.

Ring, M. J., and R. A. Plumb (2008), The response of a simplified GCM to axisymmetric forcings: Applicability of the fluctuation-dissipation theorem, J. Atmos. Sci., 65, 3880-3898.

Robinson, W. A. (1996), Does eddy feedback sustain variability in the zonal index, J. Atmos. Sci., 53, 3556-3569.

Son, S.-W., S. Lee, S. B. Feldstein, and J. E. Ten Hoeve (2008), Time scale and feedback of zonal mean flow variability, J. Atmos. Sci., 65, 935-952.

Son, S.-W., et al. (2010), The impact of stratospheric ozone on Southern Hemisphere circulation changes, J. Geophys. Res., doi:10.1029/ 2010JD014271, in press.

Strong, C., and G. Magnusdottir (2008), How Rossby wave breaking over the Pacific forces the North Atlantic Oscillation, Geophys. Res. Lett., 35 L10706, doi:10.1029/2008GL033578.

Thompson, D. W. J., and J. M. Wallace (1998), The Arctic Oscillation signature in the wintertime geopotential height and temperature fields., Geophys. Res. Lett., 25, 1297-1300.

Thompson, D. W. J., and J. M. Wallace (2000), Annular modes in the extratropical circulation: Part I. Month-to-month variability., J. Clim., 13, 1000-1016

Thompson, D. W. J., and J. M. Wallace (2001), Regional climate impacts of the Northern Hemisphere annular mode, Science, 293, 85-89, doi:10.1126/science.1058,958.

Thompson, D. W. J., J. C. Furtado, and T. G. Shepherd (2006), On the tropospheric response to anomalous stratospheric wave drag and radiative heating, J. Atmos. Sci., 63, 2616-2629.

Uppala, S. M., et al. (2005), The ERA-40 reanalysis, Q. J. R. Meteorol. Soc., 131, 2961-3012.

Vallis, G. K., and E. P. Gerber (2008), Local and hemispheric dynamics of the North Atlantic Oscillation, annular patterns, and the zonal index, Dyn. Atmos. Ocean, 44, 184-212.

Wittman, M. A. H., L. M. Polvani, R. K. Scott, and A. J. Charlton (2004), Stratospheric influence on baroclinic lifecycles and its connection to the
Artic Oscillation, Geophys. Res. Lett., 31, L16113, doi:10.1029/ 2004GL020503.

Wittman, M. A. H., A. J. Charlton, and L. M. Polvani (2005), On the meridional structure of annular modes, J. Clim., 18, 2119-2122.

Woollings, T. J., B. J. Hoskins, M. Blackburn, and P. Berrisford (2008), A new Rossby wave-breaking interpretation of the North Atlantic Oscillation, J. Atmos. Sci., 65, 609-626.

H. Akiyoshi, National Institute for Environmental Studies, 16-2 Onogawa, Tsukuba, Ibaraki 305-8506 Japan.

J. Austin, GFDL, University of Princeton, 201 Forrestal Rd., Princeton, NJ 08540-6649, USA.

M. P. Baldwin, NorthWest Research Associates, 4118 148th Ave. NE, Redmond, WA 98052, USA.

S. Bekki and M. Marchand, LATMOS, IPSL, UVSQ, UPMC, INSU, CNRS, 4 place Jussieu, Box 102, F-75231 Paris CEDEX 05, France.

P. Braesicke and J. A. Pyle, Centre for Atmospheric Science, Chemistry Department, Cambridge University, Lensfield Road, Cambridge CB2 IEW, UK.

N. Butchart and S. C. Hardiman, Met Office Hadley Centre, FitzRoy Road, Exeter EX1 3PB, UK.

M. Chipperfield and S. Dhomse, Institute for Climate and Atmospheric Science, University of Leeds, Leeds LS2 9JT, UK

M. Dameris and H. Garny, Deutsches Zentrum für Luft-und Raumfahrt, Institut für Physik der Atmosphäre, Münchner Strasse 20, D-82234 Wessling, Germany.

S. M. Frith, Atmospheric Chemistry and Dynamics Branch, NASA GSFC, Greenbelt, MD 20771, USA.

R. R. Garcia and A. Gettelman, NCAR, PO Box 3000, Boulder, CO 80307-3000, USA.

E. P. Gerber, Center for Atmosphere Ocean Science, Courant Institute of Mathematical Sciences, New York University, 251 Mercer St., New York NY 10012, USA. (gerber@cims.nyu.edu)

A. Karpechko, Finnish Meteorological Institute, PO Box 503, FI-00101 Helsinki, Finland.

O. Morgenstern and D. Smale, National Institute of Water and Atmospheric Research, State Highway 85, Central Otago, Private Bag 50061, Lauder, New Zealand.

J. E. Nielsen and S. Pawson, Global Modeling and Assimilation Office, NASA GSFC, Greenbelt, MD 20771, USA.

T. Peter and E. Rozanov, PMOD, WRC, Universitaetstrasse 16, CH-8092 Zurich, Switzerland.

D. A. Plummer and J. F. Scinocca, Canadian Centre for Climate Modelling and Analysis, Environment Canada, University of Victoria, PO Box 3065 STN CSC, Victoria, BC V8W 3V6, Canada.

T. G. Shepherd, Department of Physics, University of Toronto, $60 \mathrm{St}$. George St., Toronto, ON M5S 1A7, Canada. 